\title{
A BORG-TYPE THEOREM ASSOCIATED WITH ORTHOGONAL POLYNOMIALS ON THE UNIT CIRCLE
}

\author{
FRITZ GESZTESY AND MAXIM ZINCHENKO
}

\begin{abstract}
We prove a general Borg-type result for reflectionless unitary CMV operators $U$ associated with orthogonal polynomials on the unit circle. The spectrum of $U$ is assumed to be a connected arc on the unit circle. This extends a recent result of Simon in connection with a periodic CMV operator with spectrum the whole unit circle.

In the course of deriving the Borg-type result we also use exponential Herglotz representations of Caratheodory functions to prove an infinite sequence of trace formulas connected with the CMV operator $U$.
\end{abstract}

\section{INTRODUCTION}

The aim of this paper is to prove a Borg-type uniqueness theorem for a special class of unitary doubly infinite five-diagonal matrices. The corresponding unitary semi-infinite five-diagonal matrices were first introduced by Cantero, Moral, and Velázquez (CMV) in [8]. In [45, Sects. 4.5, 10.5], Simon introduced the corresponding notion of unitary doubly infinite five-diagonal matrices and coined the term "extended" CMV matrices. To simplify notations we will often just speak of CMV operators whether or not they are half-lattice of full-lattice operators indexed by $\mathbb{N}$ or $\mathbb{Z}$, respectively.

Before we turn to Borg-type theorems, we briefly introduce the CMV operator $U$ studied in this paper.

We denote by $\mathbb{D}$ the open unit disk in $\mathbb{C}$ and let $\alpha$ be a sequence of complex numbers in $\mathbb{D}, \alpha=\left\{\alpha_{k}\right\}_{k \in \mathbb{Z}} \subset \mathbb{D}$. The unitary CMV operator $U$ on $\ell^{2}(\mathbb{Z})$ then can be written as a special five-diagonal doubly infinite matrix in the standard basis of $\ell^{2}(\mathbb{Z})$ according to $[45$, Sects. $4.5,10.5]$ as

$$
U=\left(\begin{array}{ccccccccc}
\ddots & & \ddots & \ddots & \ddots & \ddots & & & 0 \\
& 0 & -\alpha_{0} \rho_{-1} & -\overline{\alpha_{-1}} \alpha_{0} & -\alpha_{1} \rho_{0} & \rho_{0} \rho_{1} & & 0 \\
& \rho_{-1} \rho_{0} & \overline{\alpha_{-1}} \rho_{0} & -\overline{\alpha_{0}} \alpha_{1} & \overline{\alpha_{0}} \rho_{1} & 0 & \\
& 0 & -\alpha_{2} \rho_{1} & -\overline{\alpha_{1}} \alpha_{2} & -\alpha_{3} \rho_{2} & \rho_{2} \rho_{3} & \\
& & & \rho_{1} \rho_{2} & \overline{\alpha_{1}} \rho_{2} & -\overline{\alpha_{2}} \alpha_{3} & \overline{\alpha_{2}} \rho_{3} & 0 & \\
0 & & & \ddots & & \ddots & \ddots & \ddots & \ddots
\end{array}\right) .
$$

Here the sequence of positive real numbers $\left\{\rho_{k}\right\}_{k \in \mathbb{Z}}$ is defined by

$$
\rho_{k}=\sqrt{1-\left|\alpha_{k}\right|^{2}}, \quad k \in \mathbb{Z},
$$

Date: November 15, 2004.

1991 Mathematics Subject Classification. Primary 47B36, 34A55, 47A10; Secondary 34L40.

Based upon work supported by the US National Science Foundation under Grant No. DMS0405526 . 
and terms of the form $-\overline{\alpha_{k}} \alpha_{k+1}, k \in \mathbb{Z}$, represent the diagonal entries in the infinite matrix (1.1).

The relevance of this unitary operator $U$ in $\ell^{2}(\mathbb{Z})$, more precisely, the relevance of the corresponding half-lattice CMV operator $U_{+, 0}$ in $\ell^{2}\left(\mathbb{N}_{0}\right)$ (cf. (B.17)) is derived from its intimate relationship with the trigonometric moment problem and hence with finite measures on the unit circle $\partial \mathbb{D}$. (Here $\mathbb{N}_{0}=\mathbb{N} \cup\{0\}$.) Let $\left\{\alpha_{k}\right\}_{k \in \mathbb{N}} \subset \mathbb{D}$ and define the transfer matrix

$$
S(\zeta, k)=\left(\begin{array}{cc}
\zeta & \alpha_{k} \\
\frac{\alpha_{k}}{\zeta} & 1
\end{array}\right), \quad \zeta \in \partial \mathbb{D}, k \in \mathbb{N},
$$

with spectral parameter $\zeta \in \partial \mathbb{D}$. Consider the system of difference equations

$$
\left(\begin{array}{l}
\varphi_{+}(\zeta, k) \\
\varphi_{+}^{*}(\zeta, k)
\end{array}\right)=S(\zeta, k)\left(\begin{array}{l}
\varphi_{+}(\zeta, k-1) \\
\varphi_{+}^{*}(\zeta, k-1)
\end{array}\right), \quad \zeta \in \partial \mathbb{D}, k \in \mathbb{N}
$$

with initial condition

$$
\left(\begin{array}{c}
\varphi_{+}(\zeta, 0) \\
\varphi_{+}^{*}(\zeta, 0)
\end{array}\right)=\left(\begin{array}{l}
1 \\
1
\end{array}\right), \quad \zeta \in \partial \mathbb{D}
$$

Then $\varphi_{+}(\cdot, k)$ are monic polynomials of degree $k$ and

$$
\varphi_{+}^{*}(\zeta, k)=\zeta^{k} \overline{\varphi_{+}(1 / \zeta, k)}, \quad \zeta \in \partial \mathbb{D}, k \in \mathbb{N}_{0},
$$

the reversed *-polynomial of $\varphi_{+}(\cdot, k)$, is at most of degree $k$. These polynomials were first introduced by Szegö in the 1920's in his work on the asymptotic distribution of eigenvalues of sections of Toeplitz forms [49], [50] (see also [32, Chs. 1-4], [51, Ch. XI]). Szegö's point of departure was the trigonometric moment problem and hence the theory of orthogonal polynomials on the unit circle: Given a probability measure $d \sigma_{+}$supported on an infinite set on the unit circle, find monic polynomials of degree $k$ in $\zeta=e^{i \theta}, \theta \in[0,2 \pi]$, such that

$$
\int_{0}^{2 \pi} d \sigma_{+}\left(e^{i \theta}\right) \overline{\varphi_{+}\left(e^{i \theta}, k\right)} \varphi_{+}\left(e^{i \theta}, k^{\prime}\right)=\gamma_{k}^{-2} \delta_{k, k^{\prime}}, \quad k, k^{\prime} \in \mathbb{N}_{0}
$$

where (cf. (1.2))

$$
\gamma_{k}^{2}= \begin{cases}1, & k=0 \\ \prod_{j=1}^{k} \rho_{j}^{-2}, & k \in \mathbb{N}\end{cases}
$$

One then also infers

$$
\int_{0}^{2 \pi} d \sigma_{+}\left(e^{i \theta}\right) \overline{\varphi_{+}^{*}\left(e^{i \theta}, k\right)} \varphi_{+}^{*}\left(e^{i \theta}, k^{\prime}\right)=\gamma_{k}^{-2} \delta_{k, k^{\prime}}, \quad k, k^{\prime} \in \mathbb{N}_{0}
$$

and obtains that $\varphi_{+}(\cdot, k)$ is orthogonal to $\left\{\zeta^{j}\right\}_{j=0, \ldots, k-1}$ in $L^{2}\left(\partial \mathbb{D} ; d \sigma_{+}\right)$and $\varphi_{+}^{*}(\cdot, k)$ is orthogonal to $\left\{\zeta^{j}\right\}_{j=1, \ldots, k}$ in $L^{2}\left(\partial \mathbb{D} ; d \sigma_{+}\right)$. Additional comments in this context will be provided in Remark B.6. For a detailed account of the relationship of $U_{+, 0}$ with orthogonal polynomials on the unit circle we refer to the monumental upcoming two-volume treatise by Simon [45] (see also [46] for a description of some of the principal results in [45]) and the exhaustive bibliograhy therein. For classical results on orthogonal polynomials on the unit circle we refer, for instance, to [3], [20]-[22], [32], [38], [49]-[51], [54]-[56]. More recent references relevant to the spectral theoretic content of this paper are [17], [18], [19], [27], [30], [43], [44]. Moreover, CMV operators are intimately related to a completely integrable version of the defocusing nonlinear Schrodinger equation (continuous in time but discrete 
in space), a special case of the Ablowitz-Ladik system. Relevant references in this context are, for instance, [1], [2], [16], [23], [41], [42], and the literature cited therein.

We note that $S(\zeta, k)$ in (1.3) is not the transfer matrix that leads to the halflattice CMV operator $U_{+, 0}$ in $\ell^{2}\left(\mathbb{N}_{0}\right)$ (cf. (B.15)). After a suitable change of basis introduced by Cantero, Moral, and Velázquez [8], the transfer matrix $S(\zeta, k)$ turns into $T(\zeta, k)$ as defined in (B.14).

Having introduced the notion of CMV operators, we now turn to Borg-type uniqueness theorems. From the outset, Borg-type theorems are inverse spectral theory assertions which typically prescribe a connected interval (or arc) as the spectrum of a self-adjoint (or unitary) differential or difference operator, and under a reflectionless condition imposed on the operator (one may think of a periodicity condition on the (potential) coefficients of the differential or difference operator) infers the explicit form of the coefficients of the operator in question. Typically, the form of the coefficients determined in this context is fairly simple (and usually given by constants or functions of exponential type).

Next, we briefly describe the history of Borg-type theorems relevant to this paper. In 1946, Borg [7] proved, among a variety of other inverse spectral theorems, the following result for one-dimensional Schrödinger operators. (Throughout this paper we denote by $\sigma(\cdot)$ and $\sigma_{\text {ess }}(\cdot)$ the spectrum and essential spectrum of a densely defined closed linear operator in a complex separable Hilbert space.)

Theorem $1.1([7])$.

Let $q \in L_{\mathrm{loc}}^{1}(\mathbb{R})$ be real-valued and periodic. Let $H=-\frac{d^{2}}{d x^{2}}+q$ be the associated self-adjoint Schrödinger operator in $L^{2}(\mathbb{R})$ and suppose that

$$
\sigma(H)=\left[e_{0}, \infty\right) \text { for some } e_{0} \in \mathbb{R} .
$$

Then $q$ is of the form,

$$
q(x)=e_{0} \text { for a.e. } x \in \mathbb{R} .
$$

Traditionally, uniqueness results such as Theorem 1.1 are called Borg-type theorems. However, this terminology is not uniquely adopted and hence a bit unfortunate. Indeed, inverse spectral results on finite intervals in which the potential coefficient(s) are recovered from two spectra, were also pioneered by Borg in his celebrated paper [7], and hence are also coined Borg-type theorems in the literature, see, e.g., [39], [40].

A closer examination of the proof of Theorem 1.1 in [10] shows that periodicity of $q$ is not the point for the uniqueness result (1.11). The key ingredient (besides $\sigma(H)=\left[e_{0}, \infty\right)$ and $q$ real-valued) is the fact that

$$
\text { for all } x \in \mathbb{R}, \xi(\lambda, x)=1 / 2 \text { for a.e. } \lambda \in \sigma_{\text {ess }}(h) \text {. }
$$

Here $\xi(\lambda, x)$, the argument of the boundary value $g(\lambda+i 0, x)$ of the diagonal Green's function of $H$ on the real axis (where $\left.g(z, x)=(H-z I)^{-1}(x, x)\right), z \in \mathbb{C} \backslash \sigma(h)$, $x \in \mathbb{R})$, is defined by

$$
\xi(\lambda, x)=\pi^{-1} \lim _{\varepsilon \downarrow 0} \operatorname{Im}(\ln (g(\lambda+i \varepsilon, x)) \text { for a.e. } \lambda \in \mathbb{R} \text { and all } x \in \mathbb{R} .
$$

Real-valued periodic potentials are known to satisfy (1.12), but so do certain classes of real-valued quasi-periodic and almost-periodic potentials $q$. In particular, the class of real-valued algebro-geometric finite-gap KdV potentials $q$ (a subclass of the set of real-valued quasi-periodic potentials) is a prime example satisfying (1.12) 
without necessarily being periodic. Traditionally, potentials $q$ satisfying (1.12) are called reflectionless (see [9], [10] and the references therein).

The extension of Borg's Theorem 1.1 to periodic matrix-valued Schrödinger operators was proved by Dépres [14]. A new strategy of the proof based on exponential Herglotz representations and a trace formula (cf. [25]) for such potentials, as well as the extension to reflectionless matrix-valued potentials, was obtained in [10].

The direct analog of Borg's Theorem 1.1 for periodic Jacobi operators was proved by Flaschka [15] in 1975.

Theorem $1.2([15])$.

Suppose $a=\left\{a_{k}\right\}_{k \in \mathbb{Z}}$ and $b=\left\{b_{k}\right\}_{k \in \mathbb{Z}}$ are periodic real-valued sequences in $\ell^{\infty}(\mathbb{Z})$ with the same period and $a_{k}>0, k \in \mathbb{Z}$. Let $H=a S^{+}+a^{-} S^{-}+b$ be the associated self-adjoint Jacobi operator on $\ell^{2}(\mathbb{Z})$ and suppose that

$$
\sigma(H)=\left[E_{-}, E_{+}\right] \text {for some } E_{-}<E_{+} .
$$

Then $a=\left\{a_{k}\right\}_{k \in \mathbb{Z}}$ and $b=\left\{b_{k}\right\}_{k \in \mathbb{Z}}$ are of the form,

$$
a_{k}=\left(E_{+}-E_{-}\right) / 4, \quad b_{k}=\left(E_{-}+E_{+}\right) / 2, \quad k \in \mathbb{Z} .
$$

The extension of Theorem 1.2 to reflectionless scalar Jacobi operators is due to Teschl [52, Corollary 6.3] (see also [53, Corollary 8.6]). The extension of Theorem 1.2 to matrix-valued reflectionless Jacobi operators (and a corresponding result for Dirac-type difference operators) has recently been obtained in [11].

The following very recent result of Simon is the first in connection with orthogonal polynomials on the unit circle.

Theorem 1.3 ([45], Sect. 11.14).

Suppose $\alpha=\left\{\alpha_{k}\right\}_{k \in \mathbb{Z}} \subset \mathbb{D}$ is a periodic sequence. Let $U$ be the associated unitary $C M V$ operator $(2.3)$ (cf. also (B.8)) on $\ell^{2}(\mathbb{Z})$ and suppose that

$$
\sigma(U)=\partial \mathbb{D} .
$$

Then $\alpha=\left\{\alpha_{k}\right\}_{k \in \mathbb{Z}}$ is of the form,

$$
\alpha_{k}=0, \quad k \in \mathbb{Z} .
$$

We will extend Simon's result to reflectionless Verblunsky coefficients corresponding to a CMV operator with spectrum a connected arc on the unit circle in our principal Section 5.

In Section 2 we prove an infinite sequence of trace formulas connected with CMV operators $U$ using Weyl-Titchmarsh functions (and their exponential Herglotz representations) associated with $U$. Section 3 proves certain scaling results for Schur functions associated with $U$ using a Riccati-type equation for the Verblunsky coefficients $\alpha$. The notion of reflectionless CMV operators $U$ is introduced in Section 4 and a variety of necessary conditions (many of them also sufficient) for $U$ to be reflectionless are established. In our principal Section 5 we extend Simon's Borgtype result, Theorem 1.3, from periodic to reflectionless Verblunsky coefficients, and then we prove our main new result, a Borg-type theorem for reflectionless CMV operators whose spectrum consists of a connected subarc of the unit circle $\partial \mathbb{D}$. Appendix A summarizes basic facts on Caratheodory and Schur functions relevant to this paper, and Appendix B provides some elements of Weyl-Titchmarsh theory for CMV operators on half-lattices and on $\mathbb{Z}$ (a detailed treatment of this material can be found in [27]). 


\section{Trace Formulas}

In this section we discuss trace formulas associated with the CMV operator $U$ on $\ell^{2}(\mathbb{Z})$. We freely use the notation established in Appendices A and B.

Hypothesis 2.1. Let $\alpha$ be a sequence of complex numbers such that

$$
\alpha=\left\{\alpha_{k}\right\}_{k \in \mathbb{Z}} \subset \mathbb{D} .
$$

Given a sequence $\alpha$ satisfying (2.1), we define the sequence of positive real numbers $\left\{\rho_{k}\right\}_{k \in \mathbb{Z}}$ by

$$
\rho_{k}=\sqrt{1-\left|\alpha_{k}\right|^{2}}, \quad k \in \mathbb{Z} .
$$

As discussed in (B.4)-(B.8), the unitary CMV operator $U$ on $\ell^{2}(\mathbb{Z})$ then can be written as a special five-diagonal doubly infinite matrix in the standard basis of $\ell^{2}(\mathbb{Z})($ cf. $[45$, Sects. $4.5,10.5])$ as,

$$
U=\left(\begin{array}{ccccccccc}
\ddots & & \ddots & \ddots & \ddots & \ddots & & & 0 \\
& 0 & -\alpha_{0} \rho_{-1} & -\overline{\alpha_{-1}} \alpha_{0} & -\alpha_{1} \rho_{0} & \rho_{0} \rho_{1} & & 0 \\
& \rho_{-1} \rho_{0} & \overline{\alpha_{-1}} \rho_{0} & -\overline{\alpha_{0}} \alpha_{1} & \overline{\alpha_{0}} \rho_{1} & 0 & \\
& 0 & -\alpha_{2} \rho_{1} & -\overline{\alpha_{1}} \alpha_{2} & -\alpha_{3} \rho_{2} & \rho_{2} \rho_{3} & \\
& & \rho_{1} \rho_{2} & \overline{\alpha_{1}} \rho_{2} & -\overline{\alpha_{2}} \alpha_{3} & \overline{\alpha_{2}} \rho_{3} & 0 & \\
0 & & & \ddots & & \ddots & \ddots & \ddots & \ddots
\end{array}\right) .
$$

Here terms of the form $-\overline{\alpha_{k}} \alpha_{k+1}, k \in \mathbb{Z}$, represent the diagonal entries in the infinite matrix (2.3). The half-lattice (i.e., semi-infinite) version of $U$ was first introduced by Cantero, Moral, and Velázquez [8].

Next, we recall the half-lattice Weyl-Titchmarsh functions $M_{ \pm}(\cdot, k)$ associated with $U$ (cf. (B.38)-(B.42)) and the Caratheodory function $M_{1,1}(\cdot, k)$ in (B.56). By Theorem A.3 and the fact that $M_{1,1}(0, k)=1$ by (B.59), one then obtains for the exponential Herglotz representation of $M_{1,1}(\cdot, k), k \in \mathbb{Z}$,

$$
\begin{aligned}
& -i \ln \left[i M_{1,1}(z, k)\right]=\oint_{\partial \mathbb{D}} d \mu_{0}(\zeta) \Upsilon_{1,1}(\zeta, k) \frac{\zeta+z}{\zeta-z}, \quad z \in \mathbb{D}, \\
& 0 \leq \Upsilon_{1,1}(\zeta, k) \leq \pi \text { for } \mu_{0} \text {-a.e. } \zeta \in \partial \mathbb{D} .
\end{aligned}
$$

For our present purpose it is more convenient to rewrite (2.4) in the form $(k \in \mathbb{Z})$

$$
\begin{aligned}
& \ln \left[M_{1,1}(z, k)\right]=i \oint_{\partial \mathbb{D}} d \mu_{0}(\zeta) \Xi_{1,1}(\zeta, k) \frac{\zeta+z}{\zeta-z}, \quad z \in \mathbb{D}, \\
& -\pi / 2 \leq \Xi_{1,1}(\zeta, k) \leq \pi / 2 \text { for } \mu_{0} \text {-a.e. } \zeta \in \partial \mathbb{D}
\end{aligned}
$$

where

$$
\begin{aligned}
\Xi_{1,1}(\zeta, k) & =\lim _{r \uparrow 1} \operatorname{Im}\left[\ln \left(M_{1,1}(r \zeta)\right]\right. \\
& =\Upsilon_{1,1}(\zeta, k)-(\pi / 2) \text { for } \mu_{0} \text {-a.e. } \zeta \in \partial \mathbb{D} .
\end{aligned}
$$

We note that $M_{1,1}(0, k)=1$ also implies

$$
\oint_{\partial \mathbb{D}} d \mu_{0}(\zeta) \Xi_{1,1}(\zeta, k)=0, \quad k \in \mathbb{Z}
$$

To derive trace formulas for $U$ we now expand $M_{1,1}(z, k)$ near $z=0$. Using (B.56) one obtains

$$
M_{1,1}(z, k)=\left(\delta_{k},(U+z I)(U-z I)^{-1} \delta_{k}\right)_{\ell^{2}(\mathbb{Z})}
$$




$$
\begin{aligned}
& \left.=1+2\left(\delta_{k}, z U^{*}\left(I-z U^{*}\right)^{-1}\right) \delta_{k}\right)_{\ell^{2}(\mathbb{Z})} \\
& =1+\sum_{j=1}^{\infty} M_{j}(U, k) z^{j}, \quad z \in \mathbb{D},
\end{aligned}
$$

where

$$
M_{j}(U, k)=2\left(\delta_{k},\left(U^{*}\right)^{j} \delta_{k}\right)_{\ell^{2}(\mathbb{Z})}, \quad j \in \mathbb{N}, k \in \mathbb{Z}
$$

and (2.12) represents a convergent expansion in $\mathcal{B}\left(\ell^{2}(\mathbb{Z})\right.$ ). (Here $\mathcal{B}(\mathcal{H})$ denotes the Banach space of bounded linear operators mapping the Hilbert space $\mathcal{H}$ into itself.) Explicitly, one computes

$$
M_{1}(U, k)=-2 \alpha_{k} \overline{\alpha_{k+1}}, \quad k \in \mathbb{Z} .
$$

Next, we recall the well-known fact that the convergent Taylor expansion

$$
g(z)=1+\sum_{j=1}^{\infty} c_{j} z^{j}, \quad z \in \mathbb{D}
$$

implies the absolutely convergent expansion

$$
\ln [g(z)]=\sum_{j=1}^{\infty} d_{j} z^{j}, \quad|z|<\varepsilon
$$

(for $\varepsilon=\varepsilon(g)$ sufficiently small), where $d_{j}$ can be recursivly computed via

$$
d_{1}=c_{1}, \quad d_{j}=c_{j}-\sum_{\ell=1}^{j-1}(\ell / j) c_{j-\ell} d_{\ell}, \quad j=2,3, \ldots
$$

Thus, one obtains

$$
\ln \left(M_{1,1}(z, k)\right)=\sum_{j=1}^{\infty} L_{j}(U, k) z^{j},|z| \text { sufficiently small, } k \in \mathbb{Z},
$$

where

$$
\begin{aligned}
& L_{1}(U, k)=M_{1}(U, k), \\
& L_{j}(U, k)=M_{j}(U, k)-\sum_{\ell=1}^{j-1}(\ell / j) M_{j-\ell}(U, k) L_{\ell}(U, k), \quad j=2,3, \ldots, k \in \mathbb{Z} .
\end{aligned}
$$

Theorem 2.2. Let $\alpha=\left\{\alpha_{k}\right\}_{k \in \mathbb{Z}} \subset \mathbb{D}$ and $k \in \mathbb{Z}$. Then,

$$
L_{j}(U, k)=2 i \oint_{\partial \mathbb{D}} d \mu_{0}(\zeta) \Xi_{1,1}(\zeta, k) \bar{\zeta}^{j}, \quad j \in \mathbb{N} .
$$

In particular,

$$
L_{1}(U, k)=-2 \alpha_{k} \overline{\alpha_{k+1}}=2 i \oint_{\partial \mathbb{D}} d \mu_{0}(\zeta) \Xi_{1,1}(\zeta, k) \bar{\zeta} .
$$

Proof. Let $z \in \mathbb{D}, k \in \mathbb{Z}$. Since

$$
\frac{\zeta+z}{\zeta-z}=1+2 \sum_{j=1}^{\infty}(\bar{\zeta} z)^{j}, \quad \zeta \in \partial \mathbb{D}
$$


(2.6) implies

$$
\ln \left[M_{1,1}(z, k)\right]=2 i \sum_{j=1}^{\infty} \oint_{\partial \mathbb{D}} d \mu_{0}(\zeta) \Xi_{1,1}(\zeta, k) \bar{\zeta}^{j} z^{j}, \quad|z| \text { sufficiently small. }
$$

A comparison of coefficients of $z^{j}$ in (2.17) and (2.22) then proves (2.19). (2.20) is then clear from (2.13) and (2.18).

\section{Scaling Considerations}

In this section we prove some facts about the scaling behavior of the Schur functions $\Phi_{ \pm}$and $\Phi_{1,1}$ and use that to obtain spectral results for $U$. Again we freely use the notation established in Appendices A and B.

Throughout this section we suppose that the sequence $\alpha$ satisfies Hypothesis 2.1, that is, $\alpha=\left\{\alpha_{k}\right\}_{k \in \mathbb{Z}} \subset \mathbb{D}$.

We start by recalling the Riccati-type equation (B.47) satisfied by $\Phi_{ \pm}$(cf. Remark B.14),

$$
\alpha_{k} \Phi_{ \pm}(z, k-1) \Phi_{ \pm}(z, k)-\Phi_{ \pm}(z, k-1)+z \Phi_{ \pm}(z, k)=\overline{\alpha_{k}} z, \quad z \in \mathbb{C} \backslash \partial \mathbb{D}, k \in \mathbb{Z} . \text { (3.1) }
$$

In the following it is convenient to indicate explicitly the $\alpha$-dependence of $\Phi_{ \pm}$ and $\Phi_{1,1}$ and we will thus temporarily write $\Phi_{ \pm}(z, k ; \alpha)$ and $\Phi_{1,1}(z, k ; \alpha)$, etc.

Lemma 3.1. Let $z \in \mathbb{C} \backslash \partial \mathbb{D}$ and $k \in \mathbb{Z}$. Suppose $\alpha=\left\{\alpha_{k}\right\}_{k \in \mathbb{Z}} \subset \mathbb{D}$ and assume $\left\{\gamma_{0}, \gamma_{1}\right\} \subset \partial \mathbb{D}$. Define $\beta=\left\{\gamma_{0} \gamma_{1}^{k} \alpha_{k}\right\}_{k \in \mathbb{Z}}$. Then,

$$
\begin{aligned}
\Phi_{ \pm}(z, k ; \alpha) & =\gamma_{0} \gamma_{1}^{k} \Phi_{ \pm}\left(\gamma_{1} z, k ; \beta\right), \\
\Phi_{1,1}(z, k ; \alpha) & =\Phi_{1,1}\left(\gamma_{1} z, k ; \beta\right) .
\end{aligned}
$$

Proof. We recall that

$$
\Phi_{+}(\cdot, k): \mathbb{D} \rightarrow \mathbb{D}, \quad 1 / \Phi_{-}(\cdot, k): \mathbb{D} \rightarrow \mathbb{D}, \quad k \in \mathbb{Z},
$$

are analytic, with unique Taylor coefficients at $z=0$, and hence $\Phi_{ \pm}$are the unique solutions of the Riccati-type equation (3.1) satisfying (3.4). Since the right-hand side of (3.2) also shares the mapping properties (3.4), it suffices to show that the right-hand side of (3.2) satisfies the Riccati-type equation (3.1). Multiplying

$$
\beta_{k} \Phi_{ \pm}(z, k-1 ; \beta) \Phi_{ \pm}(z, k ; \beta)-\Phi_{ \pm}(z, k-1 ; \beta)+z \Phi_{ \pm}(z, k ; \beta)-z \overline{\beta_{k}}=0
$$

by $\gamma_{0} \gamma_{1}^{k-1}$, one infers

$$
\begin{aligned}
& \beta_{k} \gamma_{0}^{-1} \gamma_{1}^{-k}\left[\gamma_{0} \gamma_{1}^{k-1} \Phi_{ \pm}(z, k-1 ; \beta)\right]\left[\gamma_{0} \gamma_{1}^{k} \Phi_{ \pm}(z, k ; \beta)\right]-\left[\gamma_{0} \gamma_{1}^{k-1} \Phi_{ \pm}(z, k-1 ; \beta)\right] \\
& \quad+z \gamma_{1}^{-1}\left[\gamma_{0} \gamma_{1}^{k} \Phi_{ \pm}(z, k ; \beta)\right]-z \gamma_{1}^{-1}\left[\overline{\beta_{k}} \overline{\gamma_{0}^{-1} \gamma_{1}^{-k}}\right]=0 .
\end{aligned}
$$

This proves (3.2). Since $\Phi_{1,1}=\Phi_{+} / \Phi_{-}$by (B.66), (3.2) implies (3.3).

Next, we also indicate the explicit $\alpha$-dependence of $U_{ \pm, k_{0}}$ and $U$ by $U_{ \pm, k_{0} ; \alpha}$ and $U_{\alpha}$, respectively. Similarly, we write $M_{ \pm}(z, k ; \alpha), M_{\ell, \ell^{\prime}}(z, k ; \alpha), \ell, \ell^{\prime}=0,1$, and $\mathcal{M}(z, k ; \alpha)$.

Corollary 3.2. Let $k_{0} \in \mathbb{Z}$. Suppose $\alpha=\left\{\alpha_{k}\right\}_{k \in \mathbb{Z}} \subset \mathbb{D}$ and assume $\left\{\gamma_{0}, \gamma_{1}\right\} \subset \partial \mathbb{D}$.

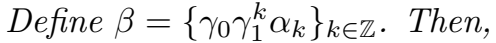

$$
\begin{aligned}
\sigma_{\mathrm{ac}}\left(U_{ \pm, k_{0} ; \alpha}\right) & =\gamma_{1}^{-1} \sigma_{\mathrm{ac}}\left(U_{ \pm, k_{0} ; \beta}\right), \\
\sigma\left(U_{\alpha}\right) & =\gamma_{1}^{-1} \sigma\left(U_{\beta}\right) .
\end{aligned}
$$

Moreover, the operators $U_{\alpha}$ and $\gamma_{1}^{-1} U_{\beta}$ are unitarily equivalent. 
Proof. Since by (B.45), $\pm \operatorname{Re}\left(M_{ \pm}\right)>0$ is equivalent to $\left|\Phi_{ \pm}^{ \pm 1}\right|<1$ and $\pm \operatorname{Re}\left(M_{ \pm}\right)>0$ is equivalent to $\pm \operatorname{Re}\left(m_{ \pm}\right)>0$ by (B.39) and (B.41), and $\left\{\gamma_{0}, \gamma_{1}\right\} \subset \partial \mathbb{D},(3.7)$ follows from (3.2), (A.14), and (A.16).

By (3.3), (A.6), (B.55), and (B.65),

$$
\begin{array}{ll}
d \Omega_{0,0}(\zeta, k ; \alpha)=d \Omega_{0,0}\left(\gamma_{1} \zeta, k ; \beta\right), & \zeta \in \partial \mathbb{D}, k \in \mathbb{Z}, \\
d \Omega_{1,1}(\zeta, k ; \alpha)=d \Omega_{1,1}\left(\gamma_{1} \zeta, k ; \beta\right), & \zeta \in \partial \mathbb{D}, k \in \mathbb{Z} .
\end{array}
$$

Applying Theorem B.18 then proves (3.8).

Finally, we prove the unitary equivalence of $U_{\alpha}$ and $\gamma_{1}^{-1} U_{\beta}$. Fix a reference point $k \in \mathbb{Z}$. By (3.3) and (B.65) one then infers

$$
M_{1,1}(z, k ; \alpha)=M_{1,1}(z, k ; \beta), \quad z \in \mathbb{C} \backslash \partial \mathbb{D}
$$

and hence also

$$
M_{0,0}(z, k ; \alpha)=M_{0,0}(z, k ; \beta), \quad z \in \mathbb{C} \backslash \partial \mathbb{D},
$$

using (B.55). Next, using (3.2) and (B.45) one computes

$$
M_{ \pm}(z, k ; \alpha)=\frac{\left(1+\gamma_{0} \gamma_{1}^{k}\right) M_{ \pm}\left(\gamma_{1} z, k ; \beta\right)+1-\gamma_{0} \gamma_{1}^{k}}{\left(1-\gamma_{0} \gamma_{1}^{k}\right) M_{ \pm}\left(\gamma_{1} z, k ; \beta\right)+1+\gamma_{0} \gamma_{1}^{k}}, \quad z \in \mathbb{C} \backslash \partial \mathbb{D} .
$$

Insertion of (3.13) into (B.60)-(B.63) then yields

$$
\begin{aligned}
& M_{0,1}(z, k ; \alpha)= \begin{cases}\gamma_{0} \gamma_{1}^{k} M_{0,1}\left(\gamma_{1} z, k ; \beta\right), & k \text { odd }, \\
\gamma_{0}^{-1} \gamma_{1}^{-k} M_{0,1}\left(\gamma_{1} z, k ; \beta\right), & k \text { even },\end{cases} \\
& M_{1,0}(z, k ; \alpha)= \begin{cases}\gamma_{0}^{-1} \gamma_{1}^{-k} M_{1,0}\left(\gamma_{1} z, k ; \beta\right), & k \text { odd }, \\
\gamma_{0} \gamma_{1}^{k} M_{1,0}\left(\gamma_{1} z, k ; \beta\right), & k \text { even }\end{cases}
\end{aligned}
$$

Thus,

$$
\mathcal{M}(z, k ; \alpha)= \begin{cases}\mathcal{A}_{k} \mathcal{M}\left(\gamma_{1} z, k ; \beta\right) \mathcal{A}_{k}^{-1}, & k \text { odd }, \\ \mathcal{A}_{k}^{-1} \mathcal{M}\left(\gamma_{1} z, k ; \beta\right) \mathcal{A}_{k}, & k \text { even, } \\ z \in \mathbb{C} \backslash \partial \mathbb{D},\end{cases}
$$

where

$$
\mathcal{A}_{k}=\left(\begin{array}{cc}
\left(\gamma_{0} \gamma_{1}^{k}\right)^{-1 / 2} & 0 \\
0 & \left(\gamma_{0} \gamma_{1}^{k}\right)^{1 / 2}
\end{array}\right), \quad k \in \mathbb{Z}
$$

Since $\gamma_{0}, \gamma_{1} \in \partial \mathbb{D}, \mathcal{M}(z, k ; \alpha)$ and $\mathcal{M}\left(\gamma_{1} z, k ; \beta\right)$ are unitarily equivalent, this implies the unitary equivalence of $U_{\alpha}$ and $\gamma_{1}^{-1} U_{\beta}$ by (B.54) and Theorems A.5 and B.18.

\section{Reflectionless Verblunsky Coefficients}

In this section we discuss a variety of equivalent conditions for the Verblunsky coefficients $\alpha$ (resp., $U$ ) to be reflectionless.

We denote by $M_{ \pm}(\zeta, k), M_{1,1}(\zeta, k), \Phi_{ \pm}(\zeta, k)$, and $\Phi_{1,1}(\zeta, k), \zeta \in \partial \mathbb{D}, k \in \mathbb{Z}$, etc., the radial limits to the unit circle of the corresponding functions,

$$
\begin{aligned}
M_{ \pm}(\zeta, k)=\lim _{r \uparrow 1} M_{ \pm}(r \zeta, k), & M_{1,1}(\zeta, k)=\lim _{r \uparrow 1} M_{1,1}(r \zeta, k), \\
\Phi_{ \pm}(\zeta, k)=\lim _{r \uparrow 1} \Phi_{ \pm}(r \zeta, k), & \Phi_{1,1}(\zeta, k)=\lim _{r \uparrow 1} \Phi_{1,1}(r \zeta, k), \quad \zeta \in \partial \mathbb{D}, k \in \mathbb{Z} .
\end{aligned}
$$


These limits are known to exist $\mu_{0}$-almost everywhere. The following definition of reflectionless Verblunsky coefficients represents the analog of reflectionless coefficients in Schrödinger, Dirac, and Jacobi operators (cf., e.g. [9], [10], [12], [13], [24], [25], [28], [29], [33], [35]-[37], [47], [48], [52], [53]).

Definition 4.1. Let $\alpha=\left\{\alpha_{k}\right\}_{k \in \mathbb{Z}} \subset \mathbb{D}$ and denote by $U$ the associated unitary CMV operator $U$ on $\ell^{2}(\mathbb{Z})$. Then $\alpha$ (resp., $U$ ) is called reflectionless, if

$$
\text { for all } k \in \mathbb{Z}, M_{+}(\zeta, k)=-\overline{M_{-}(\zeta, k)} \text { for } \mu_{0} \text {-a.e. } \zeta \in \sigma_{\text {ess }}(U) \text {. }
$$

The following result provides a variety of equivalent criteria for $\alpha$ (resp., $U$ ) to be reflectionless.

Theorem 4.2. Let $\alpha=\left\{\alpha_{k}\right\}_{k \in \mathbb{Z}} \subset \mathbb{D}$ and denote by $U$ the associated unitary $C M V$ operator $U$ on $\ell^{2}(\mathbb{Z})$. Then the following assertions $(i)-(v i)$ are equivalent:

(i) $\alpha=\left\{\alpha_{k}\right\}_{k \in \mathbb{Z}}$ is reflectionless.

(ii) Let $\gamma \in \partial \mathbb{D}$. Then $\beta=\left\{\gamma \alpha_{k}\right\}_{k \in \mathbb{Z}}$ is reflectionless.

(iii) For all $k \in \mathbb{Z}, M_{+}(\zeta, k)=-\overline{M_{-}(\zeta, k)}$ for $\mu_{0}$-a.e. $\zeta \in \sigma_{\mathrm{ess}}(U)$.

(iv) For some $k_{0} \in \mathbb{Z}, M_{+}\left(\zeta, k_{0}\right)=-\overline{M_{-}\left(\zeta, k_{0}\right)}$ for $\mu_{0}$-a.e. $\zeta \in \sigma_{\mathrm{ess}}(U)$.

(v) For all $k \in \mathbb{Z}, \Phi_{+}(\zeta, k)=1 / \overline{\Phi_{-}(\zeta, k)}$ for $\mu_{0}$-a.e. $\zeta \in \sigma_{\mathrm{ess}}(U)$.

(vi) For some $k_{0} \in \mathbb{Z}, \Phi_{+}\left(\zeta, k_{0}\right)=1 / \overline{\Phi_{-}\left(\zeta, k_{0}\right)}$ for $\mu_{0}$-a.e. $\zeta \in \sigma_{\mathrm{ess}}(U)$.

Moreover, conditions $(i)-(v i)$ imply the following equivalent assertions (vii) $-(i x)$ :

(vii) For all $k \in \mathbb{Z}, \Xi_{1,1}(\zeta, k)=0$ for $\mu_{0}$-a.e. $\zeta \in \sigma_{\mathrm{ess}}(U)$.

(viii) For all $k \in \mathbb{Z}, M_{1,1}(\zeta, k)>0$ for $\mu_{0}$-a.e. $\zeta \in \sigma_{\mathrm{ess}}(U)$.

(ix) For all $k \in \mathbb{Z}, \Phi_{1,1}(\zeta, k) \in(-1,1)$ for $\mu_{0}$-a.e. $\zeta \in \sigma_{\mathrm{ess}}(U)$.

Proof. We will prove the following diagram:

$$
\begin{gathered}
(i i) \\
\Downarrow \\
(i) \Leftrightarrow(i i i) \Leftrightarrow(v) \Leftrightarrow(v i) \Leftrightarrow(i v) \\
\Downarrow \\
(i x) \Leftrightarrow(v i i i) \Leftrightarrow(v i i)
\end{gathered}
$$

$(i)$ is equivalent to $($ iii $)$ by Definition 4.1 .

$($ iii) is equivalent to $(v)$ and $(v i)$ is equivalent to $(i v)$ by (B.44) and (B.45).

$(v) \Leftrightarrow(i i)$ : By Lemma 3.1,

$$
\Phi_{+}(z, k ; \alpha) \overline{\Phi_{-}(z, k ; \alpha)}=\Phi_{+}(z, k ; \beta) \overline{\Phi_{-}(z, k ; \beta)}, \quad z \in \mathbb{C}, k \in \mathbb{Z},
$$

hence the fact that $(i)$ is equivalent to $(v)$ implies that $(v)$ is equivalent to $(i i)$. That $(v)$ implies $(v i)$ is clear.

$(v i) \Rightarrow(v)$ : By $($ B.47),

$$
\begin{aligned}
& \Phi_{ \pm}(z, k+1)=\frac{z \overline{\alpha_{k+1}}+\Phi_{ \pm}(z, k)}{\alpha_{k+1} \Phi_{ \pm}(z, k)+z} \\
& \Phi_{ \pm}(z, k-1)=\frac{z \overline{\alpha_{k}}-z \Phi_{ \pm}(z, k)}{\alpha_{k} \Phi_{ \pm}(z, k)-1}, \quad z \in \mathbb{C} \backslash \partial \mathbb{D}, k \in \mathbb{Z} .
\end{aligned}
$$

Taking into account $(v i)$ at the point $k_{0} \in \mathbb{Z}$,

$$
\Phi_{+}\left(\zeta, k_{0}\right) \overline{\Phi_{-}\left(\zeta, k_{0}\right)}=1 \text { for } \mu_{0} \text {-a.e. } \zeta \in \sigma_{\mathrm{ess}}(U)
$$


one proves $(v i)$ at the points $k_{0} \pm 1$ as follows:

$$
\begin{aligned}
& \Phi_{+}\left(\zeta, k_{0}+1\right) \overline{\Phi_{-}\left(\zeta, k_{0}+1\right)}=\frac{\zeta \overline{\alpha_{k_{0}+1}}+\Phi_{+}\left(\zeta, k_{0}\right)}{\alpha_{k_{0}+1} \Phi_{+}\left(\zeta, k_{0}\right)+\zeta} \frac{\bar{\zeta} \alpha_{k_{0}+1}+\overline{\Phi_{-}\left(\zeta, k_{0}\right)}}{\overline{\alpha_{k_{0}+1}} \overline{\Phi_{-}\left(\zeta, k_{0}\right)}+\bar{\zeta}} \\
& \quad=\frac{1+\left|\alpha_{k_{0}+1}\right|^{2}+\bar{\zeta} \alpha_{k_{0}+1} \Phi_{+}\left(\zeta, k_{0}\right)+\zeta \overline{\alpha_{k_{0}+1}} \overline{\Phi_{-}\left(\zeta, k_{0}\right)}}{\left|\alpha_{k_{0}+1}\right|^{2}+1+\bar{\zeta} \alpha_{k_{0}+1} \Phi_{+}\left(\zeta, k_{0}\right)+\zeta \overline{\alpha_{k_{0}+1}} \overline{\Phi_{-}\left(\zeta, k_{0}\right)}} \\
& \quad=1 \text { for } \mu_{0} \text {-a.e. } \zeta \in \sigma_{\text {ess }}(U), \\
& \Phi_{+}\left(\zeta, k_{0}-1\right) \overline{\Phi_{-}\left(\zeta, k_{0}-1\right)}=|\zeta|^{2} \frac{\overline{\alpha_{k_{0}}}-\Phi_{+}\left(\zeta, k_{0}\right)}{\alpha_{k_{0}} \Phi_{+}\left(\zeta, k_{0}\right)-1} \frac{\alpha_{k_{0}}-\overline{\Phi_{-}\left(\zeta, k_{0}\right)}}{\overline{\alpha_{k_{0}}} \overline{\Phi_{-}\left(\zeta, k_{0}\right)}-1} \\
& =\frac{\left|\alpha_{k_{0}}\right|^{2}+1-\alpha_{k_{0}} \Phi_{+}\left(\zeta, k_{0}\right)-\overline{\alpha_{k_{0}}} \overline{\Phi_{-}\left(\zeta, k_{0}\right)}}{\left|\alpha_{k_{0}}\right|^{2}+1-\alpha_{k_{0}} \Phi_{+}\left(\zeta, k_{0}\right)-\overline{\alpha_{k_{0}}} \overline{\Phi_{-}\left(\zeta, k_{0}\right)}}=1 \text { for } \mu_{0} \text {-a.e. } \zeta \in \sigma_{\text {ess }}(U) .
\end{aligned}
$$

Iterating this procedure implies $(v)$.

$(i x)$ is equivalent to (viii) by (B.64) and (B.65).

(viii) is equivalent to (vii) by (2.9).

$(v) \Rightarrow(i x)$ : By $($ B.66) ,

$$
\Phi_{1,1}(z, k)=\frac{\Phi_{+}(z, k)}{\Phi_{-}(z, k)}=\frac{\Phi_{+}(z, k) \overline{\Phi_{-}(z, k)}}{\left|\Phi_{-}(z, k)\right|^{2}}, \quad z \in \mathbb{C} \backslash \partial \mathbb{D}, k \in \mathbb{Z},
$$

and hence $(v)$ implies $(i x)$.

The next result shows (the expected fact) that periodic Verblunsky coefficients are reflectionless.

Lemma 4.3. Let $\alpha=\left\{\alpha_{k}\right\}_{k \in \mathbb{Z}}$ be a sequence of periodic Verblunsky coefficients. Then $\alpha$ is reflectionless. (This applies, in particular, to $\alpha=0$.)

Proof. Let $\omega \in \mathbb{N}$ denote the period of $\alpha=\left\{\alpha_{k}\right\}_{k \in \mathbb{Z}}$. Without loss of generality we may assume $\omega$ to be even. (If $\omega$ is odd, we can consider the even period $2 \omega$.) Then,

$$
\mathfrak{M}\left(z, k_{0}\right)=\left(\begin{array}{ll}
\mathfrak{M}_{1,1}\left(z, k_{0}\right) & \mathfrak{M}_{1,2}\left(z, k_{0}\right) \\
\mathfrak{M}_{2,1}\left(z, k_{0}\right) & \mathfrak{M}_{2,2}\left(z, k_{0}\right)
\end{array}\right)=\prod_{k=1}^{\omega} T\left(z, k_{0}+k\right), \quad z \in \mathbb{C} \backslash\{0\}, k_{0} \in \mathbb{Z}
$$

represents the monodromy matrix of the CMV operator $U$ associated with the sequence $\alpha$. By $\Delta(z)$ we denote the corresponding Floquet discriminant,

$$
\Delta(z)=\frac{1}{2} \operatorname{tr}\left(\mathfrak{M}\left(z, k_{0}\right)\right), \quad z \in \mathbb{C} \backslash\{0\} .
$$

We note that $\Delta(z)$ does not depend on $k_{0}$. By (B.14) and (4.11),

$$
\begin{aligned}
& \mathfrak{M}_{1,1}\left(\zeta, k_{0}\right)=\overline{\mathfrak{M}_{2,2}\left(\zeta, k_{0}\right)}, \\
& \mathfrak{M}_{1,2}\left(\zeta, k_{0}\right)=\overline{\mathfrak{M}_{2,1}\left(\zeta, k_{0}\right)}, \quad \zeta \in \partial \mathbb{D}, k_{0} \in \mathbb{Z} .
\end{aligned}
$$

Thus, $\Delta(\zeta)=\operatorname{Re}\left(\mathfrak{M}_{1,1}\left(\zeta, k_{0}\right)\right) \in \mathbb{R}$ for all $\zeta \in \partial \mathbb{D}$. Moreover, since $\operatorname{det}\left(\mathfrak{M}\left(z, k_{0}\right)\right)=$ 1 , for all $k_{0} \in \mathbb{Z}$, the eigenvalues of $\mathfrak{M}\left(z, k_{0}\right)$ are given by

$$
\rho_{ \pm}(z)=\Delta(z) \mp \sqrt{\Delta(z)^{2}-1}, \quad z \in \mathbb{C} \backslash\{0\},
$$


where the branch of the square root is chosen such that $\left|\rho_{ \pm}(z)\right| \lessgtr 1$ for $z \in \mathbb{C} \backslash(\partial \mathbb{D} \cup$ $\{0\})$, and hence,

$$
\left(\begin{array}{l}
u_{ \pm}\left(z, k+\omega, k_{0}\right) \\
v_{ \pm}\left(z, k+\omega, k_{0}\right)
\end{array}\right)=\rho_{ \pm}(z)\left(\begin{array}{l}
u_{ \pm}\left(z, k, k_{0}\right) \\
v_{ \pm}\left(z, k, k_{0}\right)
\end{array}\right), \quad z \in \mathbb{C} \backslash\{0\}, k, k_{0} \in \mathbb{Z} .
$$

Thus, $\rho_{ \pm}$are the Floquet multipliers associated with $U$ and consequently one obtains the following characterization of the spectrum of $U$,

$$
\sigma(U)=\left\{\zeta \in \partial \mathbb{D}|| \rho_{ \pm}(\zeta) \mid=1\right\}=\{\zeta \in \partial \mathbb{D} \mid-1 \leq \Delta(\zeta) \leq 1\} .
$$

Next, assume $k$ to be even. Then (B.46) implies

$$
\begin{aligned}
\Phi_{ \pm}(z, k) & =\frac{u_{ \pm}\left(z, k, k_{0}\right)}{v_{ \pm}\left(z, k, k_{0}\right)}=\frac{u_{ \pm}\left(z, k+\omega, k_{0}\right) / \rho_{ \pm}(z)}{v_{ \pm}\left(z, k+\omega, k_{0}\right) / \rho_{ \pm}(z)} \\
& =\frac{\mathfrak{M}_{1,1}(z, k) \Phi_{ \pm}(z, k)+\mathfrak{M}_{1,2}(z, k)}{\mathfrak{M}_{2,1}(z, k) \Phi_{ \pm}(z, k)+\mathfrak{M}_{2,2}(z, k)}, \quad z \in \mathbb{C} \backslash\{0\} .
\end{aligned}
$$

It follows that

$$
\Phi_{ \pm}(z, k)=\frac{\mathfrak{M}_{1,1}(z, k)-\mathfrak{M}_{2,2}(z, k) \pm 2 \sqrt{\Delta(z)^{2}-1}}{2 \mathfrak{M}_{2,1}(z, k)}, \quad z \in \mathbb{C} \backslash\{0\},
$$

and hence, by (4.13) and (4.17),

$$
\Phi_{ \pm}(\zeta, k)=i \frac{\operatorname{Im}\left(\mathfrak{M}_{1,1}(\zeta, k)\right) \pm \sqrt{1-\operatorname{Re}\left(\mathfrak{M}_{1,1}(\zeta, k)\right)^{2}}}{\mathfrak{M}_{2,1}(\zeta, k)}, \quad \zeta \in \sigma(U) .
$$

Thus, by (4.13), (4.14), and $\operatorname{det}(\mathfrak{M}(z, k))=1$ for all $z \in \mathbb{C} \backslash\{0\}, k \in \mathbb{Z}$,

$$
\Phi_{+}(\zeta, k) \overline{\Phi_{-}(\zeta, k)}=\frac{\left|\mathfrak{M}_{1,1}(\zeta, k)\right|^{2}-1}{\left|\mathfrak{M}_{2,1}(\zeta, k)\right|^{2}}=1, \quad \zeta \in \sigma(U),
$$

and hence $\alpha$ is reflectionless by Theorem $4.2(v i)$.

We conclude this section with another result concerning the reflectionless condition (4.3) on arcs of the unit circle. It is contained in Lemma 10.11.17 in [45]. The latter is based on results in [35] (see also [31], [36]). For completeness we include the following elementary proof (which only slightly differs from that in [45] in that no $H^{p}$-arguments are involved). To fix some notation we denote by $f_{+}$and $f_{-}$a Caratheodory and anti-Caratheodory function, respectivley, and by $\varphi_{+}$and $\varphi_{-}$the corresponding Schur and anti-Schur function,

$$
\varphi_{ \pm}=\frac{f_{ \pm}-1}{f_{ \pm}+1} .
$$

Moreover, we introduce the corresponding Herglotz representations of $f_{ \pm}$(cf. (A.4), $(\mathrm{A} .5))$

$$
f_{ \pm}(z)=i c_{ \pm} \pm \oint_{\partial \mathbb{D}} d \mu_{ \pm}(\zeta) \frac{\zeta+z}{\zeta-z}, \quad z \in \mathbb{D}, c_{ \pm} \in \mathbb{R}
$$

We introduce the following notation for open arcs on the unit circle $\partial \mathbb{D}$,

$$
\operatorname{Arc}\left(\left(e^{i \theta_{1}}, e^{i \theta_{2}}\right)\right)=\left\{e^{i \theta} \in \partial \mathbb{D} \mid \theta_{1}<\theta<\theta_{2}\right\}, \quad \theta_{1} \in[0,2 \pi), \theta_{1}<\theta_{2} \leq \theta_{1}+2 \pi .
$$

An open $\operatorname{arc} A \subseteq \partial \mathbb{D}$ then either coincides with $\operatorname{Arc}\left(\left(e^{i \theta_{1}}, e^{i \theta_{2}}\right)\right)$ for some $\theta_{1} \in$ $[0,2 \pi), \theta_{1}<\theta_{2} \leq \theta_{1}+2 \pi$, or else, $A=\partial \mathbb{D}$. 
Lemma 4.4. Let $A \subseteq \partial \mathbb{D}$ be an open arc and assume that $f_{+}$(resp., $\left.f_{-}\right)$is a Caratheodory (resp., anti-Caratheodory) function satisfying the reflectionless condition (4.3) $\mu_{0}$-a.e. on $A$, that is,

$$
\lim _{r \uparrow 1}\left[f_{+}(r \zeta)+\overline{f_{-}(r \zeta)}\right]=0 \quad \mu_{0} \text {-a.e. on } A \text {. }
$$

Then,

(i) $f_{+}(\zeta)=-\overline{f_{-}(\zeta)}$ for all $\zeta \in A$.

(ii) For $z \in \mathbb{D},-\overline{f_{-}(1 / \bar{z})}$ is the analytic continuation of $f_{+}(z)$ through the arc $A$.

(iii) $d \mu_{ \pm}$are purely absolutely continuous on $A$ and

$$
\frac{d \mu_{ \pm}}{d \mu_{0}}(\zeta)=\operatorname{Re}\left(f_{+}(\zeta)\right)=-\operatorname{Re}\left(f_{-}(\zeta)\right), \quad \zeta \in A
$$

Proof. By (4.22) and

$$
\varphi_{+}(z)-\overline{1 / \varphi_{-}(z)}=\frac{-2\left[f_{+}(z)+\overline{f_{-}(z)}\right]}{\left[f_{+}(z)+1\right]\left[\overline{f_{-}(z)}-1\right]}, \quad z \in \mathbb{D},
$$

equation (4.25) is equivalent to

$$
\lim _{r \uparrow 1}\left[\varphi_{+}(r \zeta)-\overline{1 / \varphi_{-}(r \zeta)}\right]=0 \text { for } \mu_{0} \text {-a.e. } \zeta \in A \text {. }
$$

Next, introducing

$$
g_{1}(z)=\left[\varphi_{+}(z)-1 / \varphi_{-}(z)\right] / 2, \quad g_{2}(z)=\left[\varphi_{+}(z)+1 / \varphi_{-}(z)\right] /(2 i), \quad z \in \mathbb{D},
$$

then $g_{j}, j=1,2$, are Schur functions (since $z_{1}, z_{2} \in \mathbb{D}$ implies $\left(z_{1} \pm z_{2}\right) / 2 \in \mathbb{D}$ ) and hence,

$$
g_{j}+1, j=1,2, \text { are Caratheodory functions. }
$$

Moreover, by (4.28),

$$
\operatorname{Re}\left(g_{j}(\zeta)\right)=\lim _{r \uparrow 1} \operatorname{Re}\left(g_{j}(r \zeta)\right)=0 \text { for } \mu_{0} \text {-a.e. } \zeta \in A, j=1,2 .
$$

Since $g_{j}, j=1,2$, are Schur functions,

$$
\left|g_{j}(z)\right| \leq 1, \quad z \in \mathbb{D}, j=1,2,
$$

and hence the measures $d \mu_{j}$ in the Herglotz representation of $g_{j}+1, j=1,2$, are purely absolutely continuous by (A.17),

$$
d \mu_{j}=d \mu_{j, \mathrm{ac}}, \quad d \mu_{j, \mathrm{~s}}=0, \quad j=1,2 .
$$

By (A.8) and (A.14) one thus obtains

$$
\begin{aligned}
g_{j}(z)+1 & =i c_{j}+\oint_{\partial \mathbb{D}}\left[\operatorname{Re}\left(g_{j}(\zeta)\right)+1\right] d \mu_{0}(\zeta) \frac{\zeta+z}{\zeta-z}, \\
& =i c_{j}+1+\oint_{\partial \mathbb{D}} \operatorname{Re}\left(g_{j}(\zeta)\right) d \mu_{0}(\zeta) \frac{\zeta+z}{\zeta-z}, \quad z \in \mathbb{D}, j=1,2,
\end{aligned}
$$

that is,

$$
g_{j}(z)=i c_{j}+\oint_{\partial \mathbb{D}} \operatorname{Re}\left(g_{j}(\zeta)\right) d \mu_{0}(\zeta) \frac{\zeta+z}{\zeta-z}, \quad z \in \mathbb{D}, j=1,2 .
$$

By (4.31), the signed measure $\operatorname{Re}\left(g_{j}\right) d \mu_{0}$ has no support on the $\operatorname{arc} A$ and hence $g_{j}, j=1,2$, admit an analytic continuation through $A$. Moreover, using (4.35) one computes

$$
\overline{g_{j}\left(\zeta_{0}\right)}=-g_{j}\left(\zeta_{0}\right), \quad \zeta_{0} \in A, j=1,2
$$


Thus, the Schwarz symmetry principle yields

$$
g_{j}(z)=-\overline{g_{j}(1 / \bar{z})}, \quad z \in \mathbb{C} \backslash \mathbb{D}, j=1,2 .
$$

Since

$$
\varphi_{+}=g_{1}+i g_{2}, \quad 1 / \varphi_{-}=-g_{1}+i g_{2},
$$

also $\varphi_{+}$and $1 / \varphi_{-}$admit analytic continuations through the open arc $A$ and because of (4.37) (and in agreement with (4.28)) one obtains

$$
\varphi_{+}(z)=\overline{1 / \varphi_{-}(1 / \bar{z})}, \quad z \in \mathbb{C} \backslash \mathbb{D} .
$$

Thus, one computes

$$
f_{+}(z)=\frac{1+\varphi_{+}(z)}{1-\varphi_{+}(z)}=\frac{1+\overline{1 / \varphi_{-}(1 / \bar{z})}}{1-\overline{\left.1 / \varphi_{-}(1 / \bar{z})\right)}}=\frac{\overline{\varphi_{-}(1 / \bar{z})}+1}{\overline{\varphi_{-}(1 / \bar{z})}-1}=-\overline{f_{-}(1 / \bar{z})}, \quad z \in \mathbb{C} \backslash \mathbb{D} .
$$

This proves items $(i)$ and $(i i)$. In particular, $\operatorname{Re}\left(f_{ \pm}(\zeta)\right)$ exists and is finite for all $\zeta \in A$ and hence

$$
S_{\mu_{ \pm, \mathrm{s}}} \cap A=\emptyset,
$$

where $S_{\mu_{ \pm, \mathrm{s}}}$ denotes an essential support of $d \mu_{ \pm, \mathrm{s}}$. By (A.14) one thus computes

$$
\frac{d \mu_{ \pm}}{d \mu_{0}}(\zeta)=\operatorname{Re}\left(f_{+}(\zeta)\right)=-\operatorname{Re}\left(f_{-}(\zeta)\right), \quad \zeta \in A,
$$

proving item (iii).

It is perhaps worth noting that this proof is based on the elementary fact that if $g$ is any Schur function, then $g+1$ is a Caratheodory function with purely absolutely continuous measure in its Herglotz representation (cf. the first line of (4.34)). (In particular, the support of the measure in the Herglotz representation of $g+1$ equals $\partial \mathbb{D}$.) The rest are simple Schwarz symmetry considerations.

\section{The Borg-Type Theorem for CMV operators}

We recall our notation for closed arcs on the unit circle $\partial \mathbb{D}$,

$$
\operatorname{Arc}\left(\left[e^{i \theta_{1}}, e^{i \theta_{2}}\right]\right)=\left\{e^{i \theta} \in \partial \mathbb{D} \mid \theta_{1} \leq \theta \leq \theta_{2}\right\}, \quad \theta_{1} \in[0,2 \pi), \theta_{1} \leq \theta_{2} \leq \theta_{1}+2 \pi
$$

and similarly for open arcs (cf. (4.24)) and arcs open or closed at one endpoint (cf. (A.7)).

We start with a short proof of a recent result of Simon [45] in the case where $\alpha$ is a periodic sequence of Verblunsky coefficients, see Theorem 1.3. We will extend this result from the periodic to the reflectionless case.

Theorem 5.1. Let $\alpha=\left\{\alpha_{k}\right\}_{k \in \mathbb{Z}} \subset \mathbb{D}$ be a reflectionless sequence of Verblunsky coefficients. Let $U$ be the associated unitary $C M V$ operator (2.3) (cf. also (B.4)(B.7)) on $\ell^{2}(\mathbb{Z})$ and suppose that

$$
\sigma(U)=\partial \mathbb{D} .
$$

Then $\alpha=\left\{\alpha_{k}\right\}_{k \in \mathbb{Z}}$ is of the form,

$$
\alpha_{k}=0, \quad k \in \mathbb{Z} .
$$


Proof. Since by hypothesis $U$ is reflectionless, one infers from Definition 4.1 that

$$
\text { for all } k \in \mathbb{Z}, M_{+}(\zeta, k)=-\overline{M_{-}(\zeta, k)} \text { for } \mu_{0} \text {-a.e. } \zeta \in \partial \mathbb{D} \text {. }
$$

Denote by $d \omega_{ \pm}(\cdot, k)$ the measures associated with the Herglotz representation (A.4) of $M_{ \pm}(\cdot, k), k \in \mathbb{Z}$. (Of course, $d \omega_{+}=d \mu_{+}$by (B.39).) By Lemma $4.4, d \omega_{ \pm}(\cdot, k)$ are purely absolutely continuous for all $k \in \mathbb{Z}$,

$$
d \omega_{ \pm}(\cdot, k)=d \omega_{ \pm, \mathrm{ac}}(\cdot, k), \quad k \in \mathbb{Z} .
$$

Moreover, by (5.4), (5.5), and (A.14) one concludes that

$$
d \omega_{+}(\cdot, k)=d \omega_{-}(\cdot, k), \quad k \in \mathbb{Z}
$$

and hence that

$$
M_{+}(z, k)=-M_{-}(z, k), \quad z \in \mathbb{C}, k \in \mathbb{Z} .
$$

Taking $z=0$ in (5.7), and utilizing (B.40) and (B.42) then proves

$$
1=-\frac{\alpha_{k}+1}{\alpha_{k}-1}, \quad k \in \mathbb{Z}
$$

and hence (5.3) holds.

Actually, still assuming the hypotheses of Theorem 5.1, one can go a bit further: In addition to (5.3) and (5.4), (5.2) and Theorem 4.2 (vii) yield that

$$
\text { for all } k \in \mathbb{Z}, \Xi_{1,1}(\zeta, k)=0 \text { for } \mu_{0} \text {-a.e. } \zeta \in \partial \mathbb{D}
$$

and hence that

$$
M_{1,1}(z, k)=1, \quad z \in \mathbb{D}, k \in \mathbb{Z} .
$$

Moreover, (5.2), (5.4), and (B.61) yield

$$
\text { for all } k \in \mathbb{Z}, M_{ \pm}(z, k)= \pm 1, z \in \mathbb{D}, \quad d \omega_{ \pm}(\cdot, k)=d \mu_{0} .
$$

Remark 5.2. The special case where $\alpha$ is periodic and $\sigma(U)=\partial \mathbb{D}$ and thus $\alpha=0$ has originally been derived by Simon [45, Sect. 11.14] using different techniques based on Floquet theory (cf. Theorem 1.3).

The principal result of this paper then reads as follows.

Theorem 5.3. Let $\alpha=\left\{\alpha_{k}\right\}_{k \in \mathbb{Z}} \subset \mathbb{D}$ be a reflectionless sequence of Verblunsky coefficients. Let $U$ be the associated unitary CMV operator (2.3) (cf. also (B.4)(B.7)) on $\ell^{2}(\mathbb{Z})$ and suppose that the spectrum of $U$ consists of a connected arc of $\partial \mathbb{D}$,

$$
\sigma(U)=\operatorname{Arc}\left(\left[e^{i \theta_{0}}, e^{i \theta_{1}}\right]\right)
$$

with $\theta_{0} \in[0,2 \pi], \theta_{0}<\theta_{1} \leq \theta_{0}+2 \pi$, and hence $e^{i\left(\theta_{0}+\theta_{1}\right) / 2} \in \operatorname{Arc}\left(\left(e^{i \theta_{0}}, e^{i \theta_{1}}\right)\right)$. Then $\alpha=\left\{\alpha_{k}\right\}_{k \in \mathbb{Z}}$ is of the form,

$$
\alpha_{k}=\alpha_{0} g^{k}, \quad k \in \mathbb{Z},
$$

where

$$
g=-\exp \left(i\left(\theta_{0}+\theta_{1}\right) / 2\right) \text { and }\left|\alpha_{0}\right|=\cos \left(\left(\theta_{1}-\theta_{0}\right) / 4\right)
$$


Proof. By Theorem 4.2 (vii) (as a consequence of the reflectionless property of $\alpha$ ) and the fact that $M_{1,1}(\cdot, k), k \in \mathbb{Z}$, is purely imaginary on the spectral gap $\operatorname{Arc}\left(\left(e^{i \theta_{1}}, e^{i \theta_{0}+2 \pi}\right)\right)$ (since by Theorem B.18 $\left.\operatorname{supp}\left(d \Omega_{1,1}\right) \subseteq \sigma(U)\right)$ and strictly monotone as described in A.10, there exists a $\theta_{*}(k) \in\left[\theta_{1}, \theta_{0}+2 \pi\right]$ such that $\Xi_{1,1}(\cdot, k)$, $k \in \mathbb{Z}$, is of the form

$$
\Xi_{1,1}(\zeta, k)= \begin{cases}0, & \zeta \in \operatorname{Arc}\left(\left(e^{i \theta_{0}}, e^{i \theta_{1}}\right)\right), \\ \pi / 2, & \zeta \in \operatorname{Arc}\left(\left(e^{i \theta_{1}}, e^{i \theta_{*}(k)}\right)\right), \\ -\pi / 2, & \zeta \in \operatorname{Arc}\left(\left(e^{i \theta_{*}(k)}, e^{i\left(\theta_{0}+2 \pi\right)}\right)\right)\end{cases}
$$

for $\mu_{0}$-a.e. $\zeta \in \partial \mathbb{D}, k \in \mathbb{Z}$. Taking into account (2.10) then yields

$$
\begin{aligned}
0 & =\oint_{\partial \mathbb{D}} d \mu_{0}(\zeta) \Xi_{1,1}(\zeta, k)=\frac{1}{4} \oint_{\theta_{1}}^{\theta_{*}(k)} d \theta-\frac{1}{4} \oint_{\theta_{*}(k)}^{\theta_{0}+2 \pi} d \theta \\
& =\frac{1}{4}\left[2 \theta_{*}(k)-\theta_{0}-2 \pi-\theta_{1}\right], \quad k \in \mathbb{Z}
\end{aligned}
$$

and hence

$$
\theta_{*}(k)=\frac{1}{2}\left(\theta_{0}+\theta_{1}\right)+\pi, \quad k \in \mathbb{Z}
$$

is in fact $k$-independent and denoted by $\theta_{*}$ in the following. As a result, $\Xi_{1,1}(\cdot, k)=$ $\Xi_{1,1}(\cdot)$ in $(5.15)$ is also $k$-independent.

By $(2.20)$,

$$
\begin{aligned}
\alpha_{k} \overline{\alpha_{k+1}} & =-i \oint_{\partial \mathbb{D}} d \mu_{0}(\zeta) \Xi_{1,1}(\zeta) \bar{\zeta}=-i \oint_{\theta_{1}}^{\theta_{*}} \frac{\pi}{2} e^{-i t} \frac{d t}{2 \pi}+i \oint_{\theta_{*}}^{\theta_{0}+2 \pi} \frac{\pi}{2} e^{-i t} \frac{d t}{2 \pi} \\
& =-\frac{1}{4} e^{-i\left(\theta_{0}+\theta_{1}\right) / 2}\left(2+2 \cos \left(\left(\theta_{1}-\theta_{0}\right) / 2\right)\right) \\
& =-e^{-i\left(\theta_{0}+\theta_{1}\right) / 2} \cos ^{2}\left(\left(\theta_{1}-\theta_{0}\right) / 4\right), \quad k \in \mathbb{Z} .
\end{aligned}
$$

Thus, $\alpha_{k_{0}} \overline{\alpha_{k_{0}+1}}=0$ for some $k_{0} \in \mathbb{Z}$ is equivalent to $\theta_{1}=\theta_{0}+2 \pi$ and hence the assertions (5.13) and (5.14) reduce to $\alpha=0$ as in Theorem 5.1. (This is of course consistent with (5.13), (5.14), since $\left|\alpha_{0}\right|=0$ in this case.) In the case $\alpha_{k} \overline{\alpha_{k+1}} \neq 0$ for all $k \in \mathbb{Z}$, it follows from (5.18) that,

$$
\alpha_{k}=\gamma_{0} \gamma_{1}^{k} \text { and } \cdots=\left|\alpha_{1}\right|\left|\alpha_{2}\right|=\left|\alpha_{2}\right|\left|\alpha_{3}\right|=\left|\alpha_{3}\right|\left|\alpha_{4}\right|=\left|\alpha_{4}\right|\left|\alpha_{5}\right|=\cdots
$$

Hence,

$$
\alpha_{k}=\gamma_{0} \gamma_{1}^{k} \begin{cases}\left|\alpha_{1}\right|, & k \text { odd }, \\ \left|\alpha_{2}\right|, & k \text { even }\end{cases}
$$

where

$$
\left\{\gamma_{0}=\alpha_{0} /\left|\alpha_{0}\right|, \gamma_{1}=-e^{i\left(\theta_{0}+\theta_{1}\right) / 2}\right\} \subset \partial \mathbb{D} \text { and }\left|\alpha_{1}\right|\left|\alpha_{2}\right|=\cos ^{2}\left(\left(\theta_{1}-\theta_{0}\right) / 4\right) \text {. }
$$

Thus, it remains to show that $\left|\alpha_{1}\right|=\left|\alpha_{2}\right|$. We assume the contrary, $\left|\alpha_{1}\right| \neq\left|\alpha_{2}\right|$ and consider the sequence $|\alpha|=\left\{\left|\alpha_{k}\right|\right\}_{k \in \mathbb{Z}}$. Then $|\alpha|$ is a sequence of period 2 Verblunsky coefficients and by (4.12) the associated Floquet discriminant, denoted by $\Delta(\cdot ;|\alpha|)$, is given by

$$
\begin{aligned}
\Delta\left(e^{i \theta} ;|\alpha|\right) & =\frac{1}{\rho_{1} \rho_{2}}\left[\frac{e^{i \theta}+e^{-i \theta}}{2}+\operatorname{Re}\left(\left|\alpha_{1}\right| \overline{\left|\alpha_{2}\right|}\right)\right] \\
& =\frac{1}{\sqrt{1-\left|\alpha_{1}\right|^{2}} \sqrt{1-\left|\alpha_{2}\right|^{2}}}\left[\cos (\theta)+\left|\alpha_{1}\right|\left|\alpha_{2}\right|\right] .
\end{aligned}
$$


Since

$$
\sigma\left(U_{|\alpha|}\right)=\left\{e^{i \theta} \in \partial \mathbb{D} \mid-1 \leq \Delta\left(e^{i \theta} ;|\alpha|\right) \leq 1\right\}=\left\{e^{i \theta} \in \partial \mathbb{D} \mid \lambda_{-} \leq \cos (\theta) \leq \lambda_{+}\right\},
$$

where

$$
\lambda_{ \pm}=-\left|\alpha_{1}\right|\left|\alpha_{2}\right| \pm \sqrt{1-\left|\alpha_{1}\right|^{2}} \sqrt{1-\left|\alpha_{2}\right|^{2}}
$$

and $\left|\alpha_{1}\right| \neq\left|\alpha_{2}\right|$ is equivalent to $\left|\lambda_{ \pm}\right|<1, \sigma\left(U_{|\alpha|}\right)$ consists of two arcs. Taking into account (5.19), it follows from Corollary 3.2 that $\sigma\left(U_{\alpha}\right)$ should also contain two arcs which contradicts the basic hypothesis of Theorem 5.3. Thus, $\left|\alpha_{1}\right|=\left|\alpha_{2}\right|$ and (5.19) implies (5.14).

Remark 5.4. By the last part of Corollary 3.2, the phase of $\alpha_{0}$ in (5.3) is a unitary invariant and hence necessarily remains open.

\section{Appendix A. Basic Facts on Caratheodory and Schur Functions}

In this appendix we summarize a few basic properties of Caratheodory and Schur functions used throughout this manuscript.

We denote by $\mathbb{D}$ and $\partial \mathbb{D}$ the open unit disk and the unit circle in the complex plane $\mathbb{C}$,

and by

$$
\mathbb{D}=\{z \in \mathbb{C}|| z \mid<1\}, \quad \partial \mathbb{D}=\{\zeta \in \mathbb{C}|| \zeta \mid=1\}
$$

$$
\mathbb{C}_{\ell}=\{z \in \mathbb{C} \mid \operatorname{Re}(z)<0\}, \quad \mathbb{C}_{r}=\{z \in \mathbb{C} \mid \operatorname{Re}(z)>0\}
$$

the open left and right complex half-planes, respectively. Moreover, we orient the unit circle, $\partial \mathbb{D}$, counterclockwise. By a Laurent polynomial we denote a finite linear combination of terms $z^{k}, k \in \mathbb{Z}$, with complex-valued coefficients.

Definition A.1. Let $f_{ \pm}, \varphi_{+}$, and $1 / \varphi_{-}$be analytic in $\mathbb{D}$.

(i) $f_{+}$is called a Caratheodory function if $f_{+}: \mathbb{D} \rightarrow \mathbb{C}_{r}$ and $f_{-}$is called an antiCaratheodory function if $-f_{-}$is a Caratheodory function.

(ii) $\varphi_{+}$is called a Schur function if $\varphi_{+}: \mathbb{D} \rightarrow \mathbb{D} . \varphi_{-}$is called an anti-Schur function if $1 / \varphi_{-}$is a Schur function.

If $f$ and $g$ are Caratheodory functions, so are

$$
f+g, \quad 1 / f, \quad \text { and }-i \ln (i f) .
$$

Theorem A.2 ([3], Sect. 3.1; [4], Sect. 69; [45], Sect. 1.3). Let $f$ be a Caratheodory function. Then $f$ admits the Herglotz representation

$$
\begin{aligned}
& f(z)=i c+\oint_{\partial \mathbb{D}} d \mu(\zeta) \frac{\zeta+z}{\zeta-z}, \quad z \in \mathbb{D}, \\
& c=\operatorname{Im}(f(0)), \quad \oint_{\partial \mathbb{D}} d \mu(\zeta)=\operatorname{Re}(f(0))<\infty,
\end{aligned}
$$

where $d \mu$ denotes a nonnegative measure on $\partial \mathbb{D}$. The measure $d \mu$ can be reconstructed from $f$ by the formula

$$
\mu\left(\operatorname{Arc}\left(\left(e^{i \theta_{1}}, e^{i \theta_{2}}\right]\right)\right)=\lim _{\delta \downarrow 0} \lim _{r \uparrow 1} \frac{1}{2 \pi} \oint_{\theta_{1}+\delta}^{\theta_{2}+\delta} d \theta \operatorname{Re}\left(f\left(r e^{i \theta}\right)\right),
$$

where

$$
\operatorname{Arc}\left(\left(e^{i \theta_{1}}, e^{i \theta_{2}}\right]\right)=\left\{e^{i \theta} \in \partial \mathbb{D} \mid \theta_{1}<\theta \leq \theta_{2}\right\}, \quad \theta_{1} \in[0,2 \pi), \theta_{1}<\theta_{2} \leq \theta_{1}+2 \pi .
$$


Conversely, the right-hand side of (A.4) with $c \in \mathbb{R}$ and $d \mu$ a finite (nonnegative) measure on $\partial \mathbb{D}$ defines a Caratheodory function.

We note that additive nonnegative constants on the right-hand side of (A.4) can be absorbed into the measure $d \mu$ since

$$
\oint_{\partial \mathbb{D}} d \mu_{0}(\zeta) \frac{\zeta+z}{\zeta-z}=1
$$

where

$$
d \mu_{0}(\zeta)=\frac{d \theta}{2 \pi}, \quad \zeta=e^{i \theta}, \theta \in[0,2 \pi]
$$

denotes the normalized Lebesgue measure on the unit circle $\partial \mathbb{D}$.

A useful fact on Caratheodory functions $f$ is a certain monotonicity property they exhibit on open connected arcs of the unit circle away from the support of the measure $d \mu$ in the Herglotz representation (A.4). More precisely, suppose $\operatorname{Arc}\left(\left(e^{i \theta_{1}}, e^{i \theta_{2}}\right)\right) \subset(\partial \mathbb{D} \backslash \operatorname{supp}(d \mu)), \theta_{1}<\theta_{2}$, then $f$ has an analytic continuation through $\operatorname{Arc}\left(\left(e^{i \theta_{1}}, e^{i \theta_{2}}\right)\right)$ and it is purely imaginary on $\operatorname{Arc}\left(\left(e^{i \theta_{1}}, e^{i \theta_{2}}\right)\right)$. Moreover,

$$
\frac{d}{d \theta} f\left(e^{i \theta}\right)=-\frac{i}{2} \int_{[0,2 \pi] \backslash\left(\theta_{1}, \theta_{2}\right)} d \mu\left(e^{i t}\right) \frac{1}{\sin ^{2}((t-\theta) / 2)}, \quad \theta \in\left(\theta_{1}, \theta_{2}\right) .
$$

In particular,

$$
-i \frac{d}{d \theta} f\left(e^{i \theta}\right)<0, \quad \theta \in\left(\theta_{1}, \theta_{2}\right)
$$

We recall that any Caratheodory function $f$ has finite radial limits to the unit circle $\mu_{0}$-almost everywhere, that is,

$$
f(\zeta)=\lim _{r \uparrow 1} f(r \zeta) \text { exists and is finite for } \mu_{0} \text {-a.e. } \zeta \in \partial \mathbb{D} .
$$

Next, we consider the decomposition of the measure $d \mu$ in the Herglotz representation (A.4) of the Caratheodory function $f$ into its absolutely continuous and singular parts, $d \mu_{\mathrm{ac}}$ and $d \mu_{\mathrm{s}}$, respectively,

$$
d \mu=d \mu_{\mathrm{ac}}+d \mu_{\mathrm{s}} .
$$

The absolutely continuous part $d \mu_{\mathrm{ac}}$ of $d \mu$ is given by

$$
d \mu_{\mathrm{ac}}(\zeta)=\lim _{r \uparrow 1} \operatorname{Re}(f(r \zeta)) d \mu_{0}(\zeta), \quad \zeta \in \partial \mathbb{D} .
$$

The set

$$
S_{\mu_{\mathrm{ac}}}=\left\{\zeta \in \partial \mathbb{D} \mid \lim _{r \uparrow 1} \operatorname{Re}(f(r \zeta))=\operatorname{Re}(f(\zeta))>0 \text { exists finitely }\right\}
$$

is an essential support of $d \mu_{\mathrm{ac}}$ and its essential closure, $\overline{{S_{\mu_{\mathrm{ac}}}}^{e}}$, coincides with the topological support, $\operatorname{supp}\left(d \mu_{\mathrm{ac}}\right)$ (the smallest closed support), of $d \mu_{\mathrm{ac}}$,

$$
{\overline{S_{\mu_{\mathrm{ac}}}}}^{e}=\operatorname{supp}\left(d \mu_{\mathrm{ac}}\right) \text {. }
$$

Moreover, the set

$$
S_{\mu_{\mathrm{s}}}=\left\{\zeta \in \partial \mathbb{D} \mid \lim _{r \uparrow 1} \operatorname{Re}(f(r \zeta))=\infty\right\}
$$

is an essential support of the singular part $d \mu_{\mathrm{s}}$ of the measure $d \mu$, and

$$
\lim _{r \uparrow 1}(1-r) f(r \zeta)=\lim _{r \uparrow 1}(1-r) \operatorname{Re}(f(r \zeta)) \geq 0 \text { exists for all } \zeta \in \partial \mathbb{D} \text {. }
$$


In particular, $\zeta_{0} \in \partial \mathbb{D}$ is a pure point of $d \mu$ if and only if

$$
\mu\left(\left\{\zeta_{0}\right\}\right)=\lim _{r \uparrow 1}\left(\frac{1-r}{2}\right) f\left(r \zeta_{0}\right)>0 .
$$

Given a Caratheodory (resp., anti-Caratheodory) function $f_{+}$(resp. $f_{-}$) defined in $\mathbb{D}$ as in (A.4), one extends $f_{ \pm}$to all of $\mathbb{C} \backslash \partial \mathbb{D}$ by

$$
f_{ \pm}(z)=i c_{ \pm} \pm \oint_{\partial \mathbb{D}} d \mu_{ \pm}(\zeta) \frac{\zeta+z}{\zeta-z}, \quad z \in \mathbb{C} \backslash \partial \mathbb{D}, c_{ \pm} \in \mathbb{R} .
$$

In particular,

$$
f_{ \pm}(z)=-\overline{f_{ \pm}(1 / \bar{z})}, \quad z \in \mathbb{C} \backslash \overline{\mathbb{D}} .
$$

Of course, this continuation of $\left.f_{ \pm}\right|_{\mathbb{D}}$ to $\mathbb{C} \backslash \overline{\mathbb{D}}$, in general, is not an analytic continuation of $\left.f_{ \pm}\right|_{\mathbb{D}}$. With $f_{ \pm}$defined on $\mathbb{C} \backslash \partial \mathbb{D}$ by (A.6) one infers the mapping properties

$$
f_{+}: \mathbb{D} \rightarrow \mathbb{C}_{r}, \quad f_{+}: \mathbb{C} \backslash \overline{\mathbb{D}} \rightarrow \mathbb{C}_{\ell}, \quad f_{-}: \mathbb{D} \rightarrow \mathbb{C}_{\ell}, \quad f_{-}: \mathbb{C} \backslash \overline{\mathbb{D}} \rightarrow \mathbb{C}_{r} .
$$

Next, given the functions $f_{ \pm}$defined in $\mathbb{C} \backslash \partial \mathbb{D}$ as in (A.6), we introduce the functions $\varphi_{ \pm}$by

$$
\varphi_{ \pm}(z)=\frac{f_{ \pm}(z)-1}{f_{ \pm}(z)+1}, \quad z \in \mathbb{C} \backslash \partial \mathbb{D} .
$$

Then $\varphi_{ \pm}$have the mapping properties

$$
\begin{array}{ll}
\varphi_{+}: \mathbb{D} \rightarrow \mathbb{D}, \quad 1 / \varphi_{+}: \mathbb{C} \backslash \overline{\mathbb{D}} \rightarrow \mathbb{D} \quad\left(\varphi_{+}: \mathbb{C} \backslash \overline{\mathbb{D}} \rightarrow(\mathbb{C} \backslash \overline{\mathbb{D}}) \cup\{\infty\}\right), \\
\varphi_{-}: \mathbb{C} \backslash \overline{\mathbb{D}} \rightarrow \mathbb{D}, \quad 1 / \varphi_{-}: \mathbb{D} \rightarrow \mathbb{D} \quad\left(\varphi_{-}: \mathbb{D} \rightarrow(\mathbb{C} \backslash \overline{\mathbb{D}}) \cup\{\infty\}\right),
\end{array}
$$

in particular, $\left.\varphi_{+}\right|_{\mathbb{D}}\left(\right.$ resp., $\left.\left.\varphi_{-}\right|_{\mathbb{D}}\right)$ is a Schur (resp., anti-Schur) function. Moreover,

$$
f_{ \pm}(z)=\frac{1+\varphi_{ \pm}(z)}{1-\varphi_{ \pm}(z)}, \quad z \in \mathbb{C} \backslash \partial \mathbb{D} .
$$

In analogy to the exponential representation of Nevanlinna-Herglotz functions (i.e., functions analytic in the open complex upper half-plane $\mathbb{C}_{+}$with a strictly positive imaginary part on $\mathbb{C}_{+}$, cf. [5], [6], [26], [34]) one obtains the following result.

Theorem A.3. Let $f$ be a Caratheodory function. Then $-i \ln (i f)$ is a Caratheodory function and $f$ has the exponential Herglotz representation,

$$
\begin{aligned}
& -i \ln (i f(z))=i d+\oint_{\partial \mathbb{D}} d \mu_{0}(\zeta) \Upsilon(\zeta) \frac{\zeta+z}{\zeta-z}, \quad z \in \mathbb{D}, \\
& d=-\operatorname{Re}(\ln (f(0))), \quad 0 \leq \Upsilon(\zeta) \leq \pi \text { for } \mu_{0} \text {-a.e. } \zeta \in \partial \mathbb{D} .
\end{aligned}
$$

$\Upsilon$ can be reconstructed from $f$ by

$$
\begin{aligned}
\Upsilon(\zeta) & =\lim _{r \uparrow 1} \operatorname{Re}[-i \ln (i f(r \zeta))] \\
& =(\pi / 2)+\lim _{r \uparrow 1} \operatorname{Im}[\ln (f(r \zeta))] \text { for } \mu_{0} \text {-a.e. } \zeta \in \partial \mathbb{D} .
\end{aligned}
$$

Next we briefly turn to matrix-valued Caratheodory functions. We denote as usual $\operatorname{Re}(A)=\left(A+A^{*}\right) / 2, \operatorname{Im}(A)=\left(A-A^{*}\right) /(2 i)$, etc., for square matrices $A$.

Definition A.4. Let $m \in \mathbb{N}$ and $\mathcal{F}$ be an $m \times m$ matrix-valued function analytic in $\mathbb{D}$. $\mathcal{F}$ is called a Caratheodory matrix if $\operatorname{Re}(\mathcal{F}(z)) \geq 0$ for all $z \in \mathbb{D}$. 
Theorem A.5. Let $\mathcal{F}$ be an $m \times m$ Caratheodory matrix, $m \in \mathbb{N}$. Then $\mathcal{F}$ admits the Herglotz representation

$$
\begin{aligned}
& \mathcal{F}(z)=i C+\oint_{\partial \mathbb{D}} d \Omega(\zeta) \frac{\zeta+z}{\zeta-z}, \quad z \in \mathbb{D}, \\
& C=\operatorname{Im}(\mathcal{F}(0)), \quad \oint_{\partial \mathbb{D}} d \Omega(\zeta)=\operatorname{Re}(\mathcal{F}(0)),
\end{aligned}
$$

where $d \Omega$ denotes a nonnegative $m \times m$ matrix-valued measure on $\partial \mathbb{D}$. The measure $d \Omega$ can be reconstructed from $\mathcal{F}$ by the formula

$$
\begin{array}{r}
\Omega\left(\operatorname{Arc}\left(\left(e^{i \theta_{1}}, e^{i \theta_{2}}\right]\right)\right)=\lim _{\delta \downarrow 0} \lim _{r \uparrow 1} \frac{1}{2 \pi} \oint_{\theta_{1}+\delta}^{\theta_{2}+\delta} d \theta \operatorname{Re}\left(\mathcal{F}\left(r e^{i \theta}\right)\right), \\
\theta_{1} \in[0,2 \pi), \theta_{1}<\theta_{2} \leq \theta_{1}+2 \pi .
\end{array}
$$

Conversely, the right-hand side of equation (A.29) with $C=C^{*}$ and $d \Omega$ a finite nonnegative $m \times m$ matrix-valued measure on $\partial \mathbb{D}$ defines a Caratheodory matrix.

\section{Appendix B. A Summary of Weyl-Titchmarsh Theory for CMV Operators on HALF-LATTICES AND ON $\mathbb{Z}$}

We start by introducing some of the basic notations used throughout this paper. Detailed proofs of all facts in this appendix (and a lot of additional material) can be found in [27].

In the following, let $\ell^{2}(\mathbb{Z})$ be the usual Hilbert space of all square summable complex-valued sequences with scalar product $(\cdot, \cdot)_{\ell^{2}(\mathbb{Z})}$ linear in the second argument. The standard basis in $\ell^{2}(\mathbb{Z})$ is denoted by

$$
\left\{\delta_{k}\right\}_{k \in \mathbb{Z}}, \quad \delta_{k}=(\ldots, 0, \ldots, 0, \underbrace{1}_{k}, 0, \ldots, 0, \ldots)^{\top}, k \in \mathbb{Z} .
$$

For $J \subseteq \mathbb{R}$ an interval, we will identify $\ell^{2}(J \cap \mathbb{Z}) \oplus \ell^{2}(J \cap \mathbb{Z})$ and $\ell^{2}(J \cap \mathbb{Z}) \otimes \mathbb{C}^{2}$ and then use the simplified notation $\ell^{2}(J \cap \mathbb{Z})^{2}$. For simplicity, the identity operator on $\ell^{2}(J \cap \mathbb{Z})$ is abbreviated by $I$ without separately indicating its dependence on $J$.

Throughout this appendix we make the following basic assumption:

Hypothesis B.1. Let $\alpha$ be a sequence of complex numbers such that

$$
\alpha=\left\{\alpha_{k}\right\}_{k \in \mathbb{Z}} \subset \mathbb{D} .
$$

Given a sequence $\alpha$ satisfying (B.2), we define the sequence of positive real numbers $\left\{\rho_{k}\right\}_{k \in \mathbb{Z}}$ and two sequences of complex numbers with positive real parts $\left\{a_{k}\right\}_{k \in \mathbb{Z}}$ and $\left\{b_{k}\right\}_{k \in \mathbb{Z}}$ by

$$
\rho_{k}=\sqrt{1-\left|\alpha_{k}\right|^{2}}, \quad a_{k}=1+\alpha_{k}, \quad b_{k}=1-\alpha_{k}, \quad k \in \mathbb{Z} .
$$

Following Simon [45], we call $\alpha_{k}$ the Verblunsky coefficients in honor of Verblunsky's pioneering work in the theory of orthogonal polynomials on the unit circle [55], [56].

Next, we also introduce a sequence of $2 \times 2$ unitary matrices $\theta_{k}$ by

$$
\theta_{k}=\left(\begin{array}{cc}
-\alpha_{k} & \rho_{k} \\
\rho_{k} & \alpha_{k}
\end{array}\right), \quad k \in \mathbb{Z}
$$


and two unitary operators $V$ and $W$ on $\ell^{2}(\mathbb{Z})$ by their matrix representations in the standard basis of $\ell^{2}(\mathbb{Z})$ as follows,

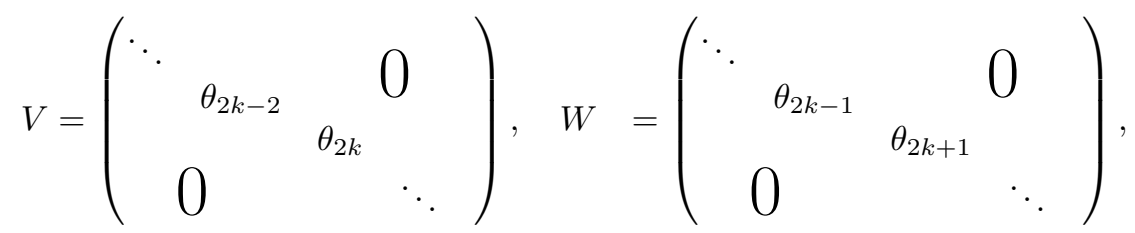

where

$$
\left(\begin{array}{cc}
V_{2 k-1,2 k-1} & V_{2 k-1,2 k} \\
V_{2 k, 2 k-1} & V_{2 k, 2 k}
\end{array}\right)=\theta_{2 k}, \quad\left(\begin{array}{cc}
W_{2 k, 2 k} & W_{2 k, 2 k+1} \\
W_{2 k+1,2 k} & W_{2 k+1,2 k+1}
\end{array}\right)=\theta_{2 k+1}, \quad k \in \mathbb{Z} .
$$

Moreover, we introduce the unitary operator $U$ on $\ell^{2}(\mathbb{Z})$ by

$$
U=V W
$$

or in matrix form, in the standard basis of $\ell^{2}(\mathbb{Z})$, by

$$
U=\left(\begin{array}{ccccccccc}
\ddots & & \ddots & \ddots & \ddots & \ddots & & & 0 \\
& 0 & -\alpha_{0} \rho_{-1} & -\overline{\alpha_{-1}} \alpha_{0} & -\alpha_{1} \rho_{0} & \rho_{0} \rho_{1} & & 0 \\
& \rho_{-1} \rho_{0} & \overline{\alpha_{-1}} \rho_{0} & -\overline{\alpha_{0}} \alpha_{1} & \overline{\alpha_{0}} \rho_{1} & 0 & \\
& 0 & -\alpha_{2} \rho_{1} & -\overline{\alpha_{1}} \alpha_{2} & -\alpha_{3} \rho_{2} & \rho_{2} \rho_{3} & \\
& & & \rho_{1} \rho_{2} & \overline{\alpha_{1}} \rho_{2} & -\overline{\alpha_{2}} \alpha_{3} & \overline{\alpha_{2}} \rho_{3} & 0 & \\
0 & & & \ddots & & \ddots & \ddots & \ddots & \ddots
\end{array}\right) .
$$

Here terms of the form $-\overline{\alpha_{k}} \alpha_{k+1}, k \in \mathbb{Z}$, represent the diagonal entries in the infinite matrix (B.8). We will call the operator $U$ on $\ell^{2}(\mathbb{Z})$ the CMV operator since (B.4)-(B.8) in the context of the semi-infinite (i.e., half-lattice) case were first obtained by Cantero, Moral, and Velázquez in [8].

Finally, let $\mathbb{U}$ denote the unitary operator on $\ell^{2}(\mathbb{Z})^{2}$ defined by

$$
\mathbb{U}=\left(\begin{array}{cc}
U & 0 \\
0 & U^{\top}
\end{array}\right)=\left(\begin{array}{cc}
V W & 0 \\
0 & W V
\end{array}\right)=\left(\begin{array}{cc}
0 & V \\
W & 0
\end{array}\right)^{2} .
$$

One observes remnants of a certain "supersymmetric" structure in $\left(\begin{array}{cc}0 & V \\ W & 0\end{array}\right)$ which is also reflected in the following result.

Lemma B.2. Let $z \in \mathbb{C} \backslash\{0\}$. Then the following items $(i)-(i v)$ are equivalent:

(i) $U u(z, \cdot)=z u(z, \cdot)$.

(ii) $\mathbb{U}\left(\begin{array}{l}u(z, \cdot) \\ v(z, \cdot)\end{array}\right)=z\left(\begin{array}{c}u(z, \cdot) \\ v(z, \cdot)\end{array}\right), \quad(W u)(z, \cdot)=z v(z, \cdot)$.

(iii) $\mathbb{U}\left(\begin{array}{c}u(z, \cdot) \\ v(z, \cdot)\end{array}\right)=z\left(\begin{array}{c}u(z, \cdot) \\ v(z, \cdot)\end{array}\right), \quad(V v)(z, \cdot)=u(z, \cdot)$.

(iv) $\left(\begin{array}{l}u(z, k) \\ v(z, k)\end{array}\right)=T(z, k)\left(\begin{array}{l}u(z, k-1) \\ v(z, k-1)\end{array}\right), \quad k \in \mathbb{Z}$, 
where the transfer matrices $T(z, k), z \in \mathbb{C} \backslash\{0\}, k \in \mathbb{Z}$, are given by

$$
T(z, k)= \begin{cases}\frac{1}{\rho_{k}}\left(\begin{array}{cc}
\alpha_{k} & z \\
1 / z & \overline{\alpha_{k}}
\end{array}\right), & k \text { odd }, \\
\frac{1}{\rho_{k}}\left(\begin{array}{cc}
\overline{\alpha_{k}} & 1 \\
1 & \alpha_{k}
\end{array}\right), & k \text { even } .\end{cases}
$$

If one sets $\alpha_{k_{0}}=e^{i s}, s \in[0,2 \pi)$, for some reference point $k_{0} \in \mathbb{Z}$, then the operator $U$ splits into a direct sum of two half-lattice operators $U_{-, k_{0}-1}^{(s)}$ and $U_{+, k_{0}}^{(s)}$ acting on $\ell^{2}\left(\left(-\infty, k_{0}-1\right] \cap \mathbb{Z}\right)$ and on $\ell^{2}\left(\left[k_{0}, \infty\right) \cap \mathbb{Z}\right)$, respectively. Explicitly, one obtains

$$
\begin{aligned}
& U=U_{-, k_{0}-1}^{(s)} \oplus U_{+, k_{0}}^{(s)} \text { in } \ell^{2}\left(\left(-\infty, k_{0}-1\right] \cap \mathbb{Z}\right) \oplus \ell^{2}\left(\left[k_{0}, \infty\right) \cap \mathbb{Z}\right) \\
& \text { if } \alpha_{k_{0}}=e^{i s}, s \in[0,2 \pi) .
\end{aligned}
$$

Similarly, one obtains $W_{-, k_{0}-1}^{(s)}, V_{-, k_{0}-1}^{(s)}$ and $W_{+, k_{0}}^{(s)}, V_{+, k_{0}}^{(s)}$ such that

$$
U_{ \pm, k_{0}}^{(s)}=V_{ \pm, k_{0}}^{(s)} W_{ \pm, k_{0}}^{(s)}
$$

For simplicity we will abbreviate

$$
U_{ \pm, k_{0}}=U_{ \pm, k_{0}}^{(s=0)}=V_{ \pm, k_{0}}^{(s=0)} W_{ \pm, k_{0}}^{(s=0)}=V_{ \pm, k_{0}} W_{ \pm, k_{0}}
$$

In addition, we introduce on $\ell^{2}\left(\left[k_{0}, \pm \infty\right) \cap \mathbb{Z}\right)^{2}$ the half-lattice operators $\mathbb{U}_{ \pm, k_{0}}^{(s)}$ by

$$
\mathbb{U}_{ \pm, k_{0}}^{(s)}=\left(\begin{array}{cc}
U_{ \pm, k_{0}}^{(s)} & 0 \\
0 & \left(U_{ \pm, k_{0}}^{(s)}\right)^{\top}
\end{array}\right)=\left(\begin{array}{cc}
V_{ \pm, k_{0}}^{(s)} W_{ \pm, k_{0}}^{(s)} & 0 \\
0 & W_{ \pm, k_{0}}^{(s)} V_{ \pm, k_{0}}^{(s)}
\end{array}\right)
$$

By $\mathbb{U}_{ \pm, k_{0}}$ we denote the half-lattice operators defined for $s=0$,

$$
\mathbb{U}_{ \pm, k_{0}}=\mathbb{U}_{ \pm, k_{0}}^{(s=0)}=\left(\begin{array}{cc}
U_{ \pm, k_{0}} & 0 \\
0 & \left(U_{ \pm, k_{0}}\right)^{\top}
\end{array}\right)=\left(\begin{array}{cc}
V_{ \pm, k_{0}} W_{ \pm, k_{0}} & 0 \\
0 & W_{ \pm, k_{0}} V_{ \pm, k_{0}}
\end{array}\right)
$$

Lemma B.3. Let $z \in \mathbb{C} \backslash\{0\}$ and $k_{0} \in \mathbb{Z}$. Consider sequences $\left\{\widehat{p}_{+}\left(z, k, k_{0}\right)\right\}_{k \geq k_{0}}$, $\left\{\widehat{r}_{+}\left(z, k, k_{0}\right)\right\}_{k \geq k_{0}}$. Then, the following items $(i)-(i i i)$ are equivalent:

(i) $\mathbb{U}_{+, k_{0}}\left(\begin{array}{c}\widehat{p}_{+}\left(z, \cdot, k_{0}\right) \\ \widehat{r}_{+}\left(z, \cdot, k_{0}\right)\end{array}\right)=z\left(\begin{array}{c}\widehat{p}_{+}\left(z, \cdot, k_{0}\right) \\ \widehat{r}_{+}\left(z, \cdot, k_{0}\right)\end{array}\right), \quad W_{+, k_{0}} \widehat{p}_{+}\left(z, \cdot, k_{0}\right)=z \widehat{r}_{+}\left(z, \cdot, k_{0}\right)$.

(ii) $\mathbb{U}_{+, k_{0}}\left(\begin{array}{c}\widehat{p}_{+}\left(z, \cdot, k_{0}\right) \\ \widehat{r}_{+}\left(z, \cdot, k_{0}\right)\end{array}\right)=z\left(\begin{array}{c}\widehat{p}_{+}\left(z, \cdot, k_{0}\right) \\ \widehat{r}_{+}\left(z, \cdot, k_{0}\right)\end{array}\right), \quad V_{+, k_{0}} \widehat{r}_{+}\left(z, \cdot, k_{0}\right)=\widehat{p}_{+}\left(z, \cdot, k_{0}\right)$.

$$
\begin{aligned}
& \left(\begin{array}{l}
\widehat{p}_{+}\left(z, k, k_{0}\right) \\
\widehat{r}_{+}\left(z, k, k_{0}\right)
\end{array}\right)=T(z, k)\left(\begin{array}{l}
\widehat{p}_{+}\left(z, k-1, k_{0}\right) \\
\widehat{r}_{+}\left(z, k-1, k_{0}\right)
\end{array}\right), \quad k>k_{0}, \\
& \text { assuming } \widehat{p}_{+}\left(z, k_{0}, k_{0}\right)= \begin{cases}z \widehat{r}_{+}\left(z, k_{0}, k_{0}\right), & k_{0} \text { odd } \\
\widehat{r}_{+}\left(z, k_{0}, k_{0}\right), & k_{0} \text { even }\end{cases}
\end{aligned}
$$


Next, consider sequences $\left\{\widehat{p}_{-}\left(z, k, k_{0}\right)\right\}_{k \leq k_{0}},\left\{\widehat{r}_{-}\left(z, k, k_{0}\right)\right\}_{k \leq k_{0}}$. Then, the following items $(i v)-(v i)$ are equivalent:

$$
\mathbb{U}_{-, k_{0}}\left(\begin{array}{c}
\widehat{p}_{-}\left(z, \cdot, k_{0}\right) \\
\widehat{r}_{-}\left(z, \cdot, k_{0}\right)
\end{array}\right)=z\left(\begin{array}{c}
\widehat{p}_{-}\left(z, \cdot, k_{0}\right) \\
\widehat{r}_{-}\left(z, \cdot, k_{0}\right)
\end{array}\right), \quad W_{-, k_{0}} \widehat{p}_{-}\left(z, \cdot, k_{0}\right)=z \widehat{r}_{-}\left(z, \cdot, k_{0}\right) .
$$

(v) $\quad \mathbb{U}_{-, k_{0}}\left(\begin{array}{c}\widehat{p}_{-}\left(z, \cdot, k_{0}\right) \\ \widehat{r}_{-}\left(z, \cdot, k_{0}\right)\end{array}\right)=z\left(\begin{array}{c}\widehat{p}_{-}\left(z, \cdot, k_{0}\right) \\ \widehat{r}_{-}\left(z, \cdot, k_{0}\right)\end{array}\right), \quad V_{-, k_{0}} \widehat{r}_{-}\left(z, \cdot, k_{0}\right)=\widehat{p}_{-}\left(z, \cdot, k_{0}\right)$.

(vi) $\quad\left(\begin{array}{l}\widehat{p}_{-}(z, k-1), k_{0} \\ \widehat{r}_{-}\left(z, k-1, k_{0}\right)\end{array}\right)=T(z, k)^{-1}\left(\begin{array}{c}\widehat{p}_{-}\left(z, k, k_{0}\right) \\ \widehat{r}_{-}\left(z, k, k_{0}\right)\end{array}\right), \quad k \leq k_{0}$,

$$
\text { assuming } \widehat{p}_{-}\left(z, k_{0}, k_{0}\right)= \begin{cases}-\widehat{r}_{-}\left(z, k_{0}, k_{0}\right), & k_{0} \text { odd }, \\ -z \widehat{r}_{-}\left(z, k_{0}, k_{0}\right), & k_{0} \text { even } .\end{cases}
$$

In the following, we denote by $\left(\begin{array}{c}p_{+}\left(z, k, k_{0}\right) \\ r_{+}\left(z, k, k_{0}\right)\end{array}\right)_{k \geq k_{0}}$ and $\left(\begin{array}{c}q_{+}\left(z, k, k_{0}\right) \\ s_{+}\left(z, k, k_{0}\right)\end{array}\right)_{k \geq k_{0}}, z \in \mathbb{C} \backslash\{0\}$, two linearly independent solutions of (B.22) with the following initial conditions:

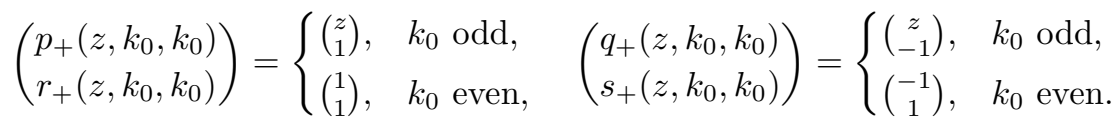

Similarly, we denote by $\left(\begin{array}{c}p_{-}\left(z, k, k_{0}\right) \\ r_{-}\left(z, k, k_{0}\right)\end{array}\right)_{k \leq k_{0}}$ and $\left(\begin{array}{c}q_{-}\left(z, k, k_{0}\right) \\ s_{-}\left(z, k, k_{0}\right)\end{array}\right)_{k \leq k_{0}}, z \in \mathbb{C} \backslash\{0\}$, two linearly independent solutions of (B.26) with the following initial conditions:

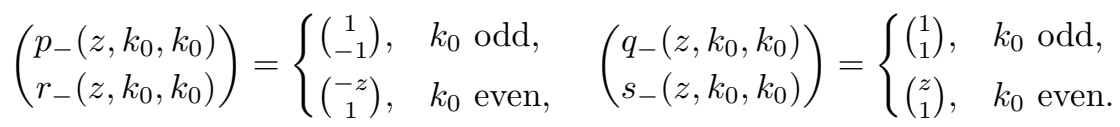

Using (B.13) one extends $\left(\begin{array}{c}p_{+}\left(z, k, k_{0}\right) \\ r_{+}\left(z, k, k_{0}\right)\end{array}\right)_{k \geq k_{0}},\left(\begin{array}{c}q_{+}\left(z, k, k_{0}\right) \\ s_{+}\left(z, k, k_{0}\right)\end{array}\right)_{k \geq k_{0}}, z \in \mathbb{C} \backslash\{0\}$, to $k<$ $k_{0}$. In the same manner, one extends $\left(\begin{array}{c}p_{-}\left(z, k, k_{0}\right) \\ r_{-}\left(z, k, k_{0}\right)\end{array}\right)_{k \leq k_{0}}$ and $\left(\begin{array}{c}q_{-}\left(z, k, k_{0}\right) \\ s_{-}\left(z, k, k_{0}\right)\end{array}\right)_{k \leq k_{0}}, z \in$ $\mathbb{C} \backslash\{0\}$, to $k>k_{0}$. These extensions will be denoted by $\left(\begin{array}{c}p_{ \pm}\left(z, k, k_{0}\right) \\ r_{ \pm}\left(z, k, k_{0}\right)\end{array}\right){ }_{k \in \mathbb{Z}}$ and $\left(\begin{array}{c}q_{ \pm}\left(z, k, k_{0}\right) \\ s_{ \pm}\left(z, k, k_{0}\right)\end{array}\right)_{k \in \mathbb{Z}}$. Moreover, it follows from (B.13) that $p_{ \pm}\left(z, k, k_{0}\right), q_{ \pm}\left(z, k, k_{0}\right)$, $r_{ \pm}\left(z, k, k_{0}\right)$, and $s_{ \pm}\left(z, k, k_{0}\right), k, k_{0} \in \mathbb{Z}$, are Laurent polynomials in $z$.

Remark B.4. By Lemma B.3, $\left(\begin{array}{c}p_{ \pm}\left(z, k, k_{0}\right) \\ r_{ \pm}\left(z, k, k_{0}\right)\end{array}\right)_{k \gtreqless k_{0}}, z \in \mathbb{C} \backslash\{0\}, k_{0} \in \mathbb{Z}$, are generalized eigenvectors of the operators $\mathbb{U}_{ \pm, k_{0}}$. Moreover, by Lemma B.2, $\left(\begin{array}{l}p_{ \pm}\left(z, k, k_{0}\right) \\ r_{ \pm}\left(z, k, k_{0}\right)\end{array}\right)_{k \in \mathbb{Z}}$ and $\left(\begin{array}{c}q_{ \pm}\left(z, k, k_{0}\right) \\ s_{ \pm}\left(z, k, k_{0}\right)\end{array}\right)_{k \in \mathbb{Z}}, z \in \mathbb{C} \backslash\{0\}, k_{0} \in \mathbb{Z}$, are generalized eigenvectors of $\mathbb{U}$.

Lemma B.5. Let $k_{0} \in \mathbb{Z}$. Then the sets of Laurent polynomials $\left\{p_{ \pm}\left(\cdot, k, k_{0}\right)\right\}_{k_{\gtrless}} k_{0}$ and $\left\{r_{ \pm}\left(\cdot, k, k_{0}\right)\right\}_{k^{2} k_{0}}$ form complete orthonormal systems in $L^{2}\left(\partial \mathbb{D} ; d \mu_{ \pm}\left(\cdot, k_{0}\right)\right)$, where

$$
d \mu_{ \pm}\left(\zeta, k_{0}\right)=d\left(\delta_{k_{0}}, E_{U_{ \pm, k_{0}}}(\zeta) \delta_{k_{0}}\right), \quad \zeta \in \partial \mathbb{D}
$$

and $d E_{U_{ \pm, k_{0}}}(\cdot)$ denote the operator-valued spectral measures of the operators $U_{ \pm, k_{0}}$,

$$
U_{ \pm, k_{0}}=\oint_{\partial \mathbb{D}} d E_{U_{ \pm, k_{0}}}(\zeta) \zeta
$$


We note that the measures $d \mu_{ \pm}\left(\cdot, k_{0}\right), k_{0} \in \mathbb{Z}$, are not only nonnegative but also supported on an infinite set.

Remark B.6. In connection with our introductory remarks in (1.3)-(1.9) we note that $d \sigma_{+}=d \mu_{+}(\cdot, 0)$ and that in accordance with [45, Proposition 4.2.2]

$$
\begin{aligned}
& p_{+}(\zeta, k, 0)= \begin{cases}\gamma_{k} \zeta^{-(k-1) / 2} \varphi_{+}(\zeta, k), & k \text { odd }, \\
\gamma_{k} \zeta^{-k / 2} \varphi_{+}^{*}(\zeta, k), & k \text { even, }\end{cases} \\
& r_{+}(\zeta, k, 0)=\left\{\begin{array}{ll}
\gamma_{k} \zeta^{-(k+1) / 2} \varphi_{+}^{*}(\zeta, k), & k \text { odd, } \\
\gamma_{k} \zeta^{-k / 2} \varphi_{+}(\zeta, k), & k \text { even } ;
\end{array} \quad \zeta \in \partial \mathbb{D} .\right.
\end{aligned}
$$

Corollary B.7. Let $k_{0} \in \mathbb{Z}$. Then the half-lattice $C M V$ operators $U_{ \pm, k_{0}}$ are unitarily equivalent to the operators of multiplication by the function id (where $i d(\zeta)=\zeta$, $\zeta \in \partial \mathbb{D})$ on $L^{2}\left(\partial \mathbb{D} ; d \mu_{ \pm}\left(\cdot, k_{0}\right)\right)$. In particular,

$$
\sigma\left(U_{ \pm, k_{0}}\right)=\operatorname{supp}\left(d \mu_{ \pm}\left(\cdot, k_{0}\right)\right)
$$

and the spectrum of $U_{ \pm, k_{0}}$ is simple.

Lemma B.8. Let $k_{0} \in \mathbb{Z}$. Then the sets of two-dimensional Laurent polynomials $\left(\begin{array}{c}p_{ \pm}\left(z, k, k_{0}\right) \\ r_{ \pm}\left(z, k, k_{0}\right)\end{array}\right)_{k \gtreqless k_{0}}$ and $\left(\begin{array}{c}q_{ \pm}\left(z, k, k_{0}\right) \\ s_{ \pm}\left(z, k, k_{0}\right)\end{array}\right)_{k \gtreqless k_{0}}$ satisfy the relation

$$
\left(\begin{array}{l}
q_{ \pm}\left(z, \cdot, k_{0}\right) \\
s_{ \pm}\left(z, \cdot, k_{0}\right)
\end{array}\right)+m_{ \pm}\left(z, k_{0}\right)\left(\begin{array}{l}
p_{ \pm}\left(z, \cdot, k_{0}\right) \\
r_{ \pm}\left(z, \cdot, k_{0}\right)
\end{array}\right) \in \ell^{2}\left(\left[k_{0}, \pm \infty\right) \cap \mathbb{Z}\right)^{2}, \quad z \in \mathbb{C} \backslash(\partial \mathbb{D} \cup\{0\}),
$$

where the coefficients $m_{ \pm}\left(z, k_{0}\right)$ are given by

$$
\begin{aligned}
m_{ \pm}\left(z, k_{0}\right) & = \pm\left(\delta_{k_{0}},\left(U_{ \pm, k_{0}}+z I\right)\left(U_{ \pm, k_{0}}-z I\right)^{-1} \delta_{k_{0}}\right)_{\ell^{2}(\mathbb{Z})} \\
& = \pm \oint_{\partial \mathbb{D}} d \mu_{ \pm}\left(\zeta, k_{0}\right) \frac{\zeta+z}{\zeta-z}, \quad z \in \mathbb{C} \backslash \partial \mathbb{D}
\end{aligned}
$$

with

$$
m_{ \pm}\left(0, k_{0}\right)= \pm \oint_{\partial \mathbb{D}} d \mu_{ \pm}\left(\zeta, k_{0}\right)= \pm 1
$$

Lemma B.9. Let $k_{0} \in \mathbb{Z}$. Then relation (B.34) uniquely determines the functions $m_{ \pm}\left(\cdot, k_{0}\right)$ on $\mathbb{C} \backslash \partial \mathbb{D}$.

Theorem B.10. Let $k_{0} \in \mathbb{Z}$. Then there exist unique functions $M_{ \pm}\left(\cdot, k_{0}\right)$ such that

$$
\begin{gathered}
\left(\begin{array}{l}
u_{ \pm}\left(z, \cdot, k_{0}\right) \\
v_{ \pm}\left(z, \cdot, k_{0}\right)
\end{array}\right)=\left(\begin{array}{c}
q_{+}\left(z, \cdot, k_{0}\right) \\
s_{+}\left(z, \cdot, k_{0}\right)
\end{array}\right)+M_{ \pm}\left(z, k_{0}\right)\left(\begin{array}{c}
p_{+}\left(z, \cdot, k_{0}\right) \\
r_{+}\left(z, \cdot, k_{0}\right)
\end{array}\right) \in \ell^{2}\left(\left[k_{0}, \pm \infty\right) \cap \mathbb{Z}\right)^{2} \\
z \in \mathbb{C} \backslash(\partial \mathbb{D} \cup\{0\}) .
\end{gathered}
$$

We will call $u_{ \pm}\left(z, \cdot, k_{0}\right)$ (resp., $v_{ \pm}\left(z, \cdot, k_{0}\right)$ ) Weyl-Titchmarsh solutions of $U$ (resp., $\left.U^{\top}\right)$. Similarly, we will call $m_{ \pm}\left(z, k_{0}\right)$ as well as $M_{ \pm}\left(z, k_{0}\right)$ the half-lattice Weyl-Titchmarsh $m$-functions associated with $U_{ \pm, k_{0}}$. (See also [44] for a comparison of various alternative notions of Weyl-Titchmarsh $m$-functions for $U_{+, k_{0}}$.)

One verifies that

$$
\begin{aligned}
& M_{+}\left(z, k_{0}\right)=m_{+}\left(z, k_{0}\right), \quad z \in \mathbb{C} \backslash \partial \mathbb{D}, \\
& M_{+}\left(0, k_{0}\right)=1,
\end{aligned}
$$




$$
\begin{aligned}
& M_{-}\left(z, k_{0}\right)=\frac{\operatorname{Re}\left(a_{k_{0}}\right)+i \operatorname{Im}\left(b_{k_{0}}\right) m_{-}\left(z, k_{0}-1\right)}{i \operatorname{Im}\left(a_{k_{0}}\right)+\operatorname{Re}\left(b_{k_{0}}\right) m_{-}\left(z, k_{0}-1\right)}, \quad z \in \mathbb{C} \backslash \partial \mathbb{D}, \\
& M_{-}\left(0, k_{0}\right)=\frac{\alpha_{k_{0}}+1}{\alpha_{k_{0}}-1} .
\end{aligned}
$$

In particular, one infers that $M_{ \pm}$are analytic at $z=0$.

Lemma B.11. Let $k \in \mathbb{Z}$. Then the functions $\left.M_{+}(\cdot, k)\right|_{\mathbb{D}}\left(\right.$ resp., $\left.\left.M_{-}(\cdot, k)\right|_{\mathbb{D}}\right)$ are Caratheodory (resp., anti-Caratheodory) functions. Moreover, $M_{ \pm}$satisfy the following Riccati-type equation

$$
\begin{aligned}
& \left(z \overline{b_{k}}-b_{k}\right) M_{ \pm}(z, k-1) M_{ \pm}(z, k)+\left(z \overline{b_{k}}+b_{k}\right) M_{ \pm}(z, k)-\left(z \overline{a_{k}}+a_{k}\right) M_{ \pm}(z, k-1) \\
& \quad=z \overline{a_{k}}-a_{k}, \quad z \in \mathbb{C} \backslash \partial \mathbb{D} .
\end{aligned}
$$

In addition, we introduce the functions $\Phi_{ \pm}(\cdot, k), k \in \mathbb{Z}$, by

$$
\Phi_{ \pm}(z, k)=\frac{M_{ \pm}(z, k)-1}{M_{ \pm}(z, k)+1}, \quad z \in \mathbb{C} \backslash \partial \mathbb{D} .
$$

One then verifies,

$$
M_{ \pm}(z, k)=\frac{1+\Phi_{ \pm}(z, k)}{1-\Phi_{ \pm}(z, k)}, \quad z \in \mathbb{C} \backslash \partial \mathbb{D} .
$$

Lemma B.12. Let $z \in \mathbb{C} \backslash(\partial \mathbb{D} \cup\{0\}), k_{0}, k \in \mathbb{Z}$. Then the functions $\Phi_{ \pm}(\cdot, k)$ satisfy the following equalities

$$
\Phi_{ \pm}(z, k)= \begin{cases}z \frac{v_{ \pm}\left(z, k, k_{0}\right)}{u_{ \pm}\left(z, k, k_{0}\right)}, & k \text { odd }, \\ \frac{u_{ \pm}\left(z, k, k_{0}\right)}{v_{ \pm}\left(z, k, k_{0}\right)}, & k \text { even },\end{cases}
$$

where $u_{ \pm}\left(\cdot, k, k_{0}\right)$ and $v_{ \pm}\left(\cdot, k, k_{0}\right)$ are the polynomials defined in (B.38).

Lemma B.13. Let $k \in \mathbb{Z}$. Then the functions $\left.\Phi_{+}(\cdot, k)\right|_{\mathbb{D}}\left(\right.$ resp., $\left.\left.\Phi_{-}(\cdot, k)\right|_{\mathbb{D}}\right)$ are Schur (resp., anti-Schur) functions. Moreover, $\Phi_{ \pm}$satisfy the following Riccati-type equation

$$
\alpha_{k} \Phi_{ \pm}(z, k-1) \Phi_{ \pm}(z, k)-\Phi_{ \pm}(z, k-1)+z \Phi_{ \pm}(z, k)=\overline{\alpha_{k}} z, \quad z \in \mathbb{C} \backslash \partial \mathbb{D}, k \in \mathbb{Z} .
$$

Remark B.14. (i) In the special case $\alpha=\left\{\alpha_{k}\right\}_{k \in \mathbb{Z}}=0$, one obtains

$$
M_{ \pm}(z, k)= \pm 1, \quad \Phi_{+}(z, k)=0, \quad 1 / \Phi_{-}(z, k)=0, \quad z \in \mathbb{C}, k \in \mathbb{Z} .
$$

Thus, strictly speaking, one should always consider $1 / \Phi_{-}$rather than $\Phi_{-}$and hence refer to the Riccati-type equation of $1 / \Phi_{-}$,

$$
\overline{\alpha_{k}} z \frac{1}{\Phi_{-}(z, k-1)} \frac{1}{\Phi_{-}(z, k)}+\frac{1}{\Phi_{-}(z, k)}-z \frac{1}{\Phi_{-}(z, k-1)}=\alpha_{k}, \quad z \in \mathbb{C} \backslash \partial \mathbb{D}, k \in \mathbb{Z},
$$

rather than that of $\Phi_{-}$, etc. For simplicity of notation, we will often avoid this distinction between $\Phi_{-}$and $1 / \Phi_{-}$and usually just invoke $\Phi_{-}$whenever confusions are unlikely.

(ii) We note that $M_{ \pm}(z, k)$ and $\Phi_{ \pm}(z, k), z \in \partial \mathbb{D}, k \in \mathbb{Z}$, have nontangential limits to $\partial \mathbb{D} \mu_{0}$-a.e. In particular, the Riccati-type equations (B.43), (B.47), and (B.49) extend to $\partial \mathbb{D} \mu_{0}$-a.e. 
Lemma B.15. Let $z \in \mathbb{C} \backslash(\partial \mathbb{D} \cup\{0\})$ and fix $k_{0}, k_{1} \in \mathbb{Z}$. Then the resolvent $(U-z I)^{-1}$ of the unitary $C M V$ operator $U$ on $\ell^{2}(\mathbb{Z})$ is given in terms of its matrix representation in the standard basis of $\ell^{2}(\mathbb{Z})$ by

$$
\begin{aligned}
& (U-z I)^{-1}\left(k, k^{\prime}\right)=\frac{(-1)^{k_{1}+1}}{z W\left(\left(\begin{array}{l}
u_{+}\left(z, k_{1}, k_{0}\right) \\
v_{+}\left(z, k_{1}, k_{0}\right)
\end{array}\right),\left(\begin{array}{l}
u_{-}\left(z, k_{1}, k_{0}\right) \\
v_{-}\left(z, k_{1}, k_{0}\right)
\end{array}\right)\right)} \\
& \quad \times\left\{\begin{array}{l}
u_{-}\left(z, k, k_{0}\right) v_{+}\left(z, k^{\prime}, k_{0}\right), \quad k<k^{\prime} \text { and } k=k^{\prime} \text { odd }, \\
v_{-}\left(z, k^{\prime}, k_{0}\right) u_{+}\left(z, k, k_{0}\right), \quad k^{\prime}<k \text { and } k=k^{\prime} \text { even, }
\end{array} \quad k, k^{\prime} \in \mathbb{Z},\right.
\end{aligned}
$$

where

$$
\begin{gathered}
W\left(\left(\begin{array}{l}
u_{+}\left(z, k_{1}, k_{0}\right) \\
v_{+}\left(z, k_{1}, k_{0}\right)
\end{array}\right),\left(\begin{array}{l}
u_{-}\left(z, k_{1}, k_{0}\right) \\
v_{-}\left(z, k_{1}, k_{0}\right)
\end{array}\right)\right)=\operatorname{det}\left(\left(\begin{array}{lll}
u_{+}\left(z, k_{1}, k_{0}\right) & u_{-}\left(z, k_{1}, k_{0}\right) \\
v_{+}\left(z, k_{1}, k_{0}\right) & v_{-}\left(z, k_{1}, k_{0}\right)
\end{array}\right)\right) \\
=(-1)^{k_{1}}\left[M_{+}\left(z, k_{0}\right)-M_{-}\left(z, k_{0}\right)\right]\left\{\begin{array}{lll}
2 z, & k_{0} \text { odd }, & k_{0}, k_{1} \in \mathbb{Z} . \\
2, & k_{0} \text { even, }
\end{array}\right.
\end{gathered}
$$

Moreover, since $0 \in \mathbb{C} \backslash \sigma(U)$, (B.50) analytically extends to $z=0$.

Next, we briefly turn to Weyl-Titchmarsh theory for CMV operators on $\mathbb{Z}$. We denote by $d \Omega(\cdot, k), k \in \mathbb{Z}$, the $2 \times 2$ matrix-valued measure,

$$
\begin{aligned}
d \Omega(\zeta, k) & =d\left(\begin{array}{cc}
\Omega_{0,0}(\zeta, k) & \Omega_{0,1}(\zeta, k) \\
\Omega_{1,0}(\zeta, k) & \Omega_{1,1}(\zeta, k)
\end{array}\right) \\
& =d\left(\begin{array}{cc}
\left(\delta_{k-1}, E_{U}(\zeta) \delta_{k-1}\right)_{\ell^{2}(\mathbb{Z})} & \left(\delta_{k-1}, E_{U}(\zeta) \delta_{k}\right)_{\ell^{2}(\mathbb{Z})} \\
\left(\delta_{k}, E_{U}(\zeta) \delta_{k-1}\right)_{\ell^{2}(\mathbb{Z})} & \left(\delta_{k}, E_{U}(\zeta) \delta_{k}\right)_{\ell^{2}(\mathbb{Z})}
\end{array}\right), \quad \zeta \in \partial \mathbb{D},
\end{aligned}
$$

where $d E_{U}(\cdot)$ denotes the operator-valued spectral measure of the unitary CVM operator $U$ on $\ell^{2}(\mathbb{Z})$,

$$
U=\oint_{\partial \mathbb{D}} d E_{U}(\zeta) \zeta
$$

We also introduce the $2 \times 2$ matrix-valued function $\mathcal{M}(\cdot, k), k \in \mathbb{Z}$, by

$$
\begin{aligned}
\mathcal{M} & (z, k)=\left(\begin{array}{ll}
M_{0,0}(z, k) & M_{0,1}(z, k) \\
M_{1,0}(z, k) & M_{1,1}(z, k)
\end{array}\right) \\
& =\left(\begin{array}{cc}
\left(\delta_{k-1},(U+z I)(U-z I)^{-1} \delta_{k-1}\right)_{\ell^{2}(\mathbb{Z})} & \left(\delta_{k-1},(U+z I)(U-z I)^{-1} \delta_{k}\right)_{\ell^{2}(\mathbb{Z})} \\
\left(\delta_{k},(U+z I)(U-z I)^{-1} \delta_{k-1}\right)_{\ell^{2}(\mathbb{Z})} & \left(\delta_{k},(U+z I)(U-z I)^{-1} \delta_{k}\right)_{\ell^{2}(\mathbb{Z})}
\end{array}\right) \\
& =\oint_{\partial \mathbb{D}} d \Omega(\zeta, k) \frac{\zeta+z}{\zeta-z}, \quad z \in \mathbb{C} \backslash \partial \mathbb{D} .
\end{aligned}
$$

We note that

$$
M_{0,0}(\cdot, k+1)=M_{1,1}(\cdot, k), \quad k \in \mathbb{Z}
$$

and

$$
\begin{aligned}
M_{1,1}(z, k) & =\left(\delta_{k},(U+z I)(U-z I)^{-1} \delta_{k}\right)_{\ell^{2}(\mathbb{Z})} \\
& =\oint_{\partial \mathbb{D}} d \Omega_{1,1}(\zeta, k) \frac{\zeta+z}{\zeta-z}, \quad z \in \mathbb{C} \backslash \partial \mathbb{D}, k \in \mathbb{Z},
\end{aligned}
$$

where

$$
d \Omega_{1,1}(\zeta, k)=d\left(\delta_{k}, E_{U}(\zeta) \delta_{k}\right)_{\ell^{2}(\mathbb{Z})}, \quad \zeta \in \partial \mathbb{D} .
$$


Thus, $\left.M_{0,0}\right|_{\mathbb{D}}$ and $\left.M_{1,1}\right|_{\mathbb{D}}$ are Caratheodory functions. Moreover, by (B.56) one infers that

$$
M_{1,1}(0, k)=1, \quad k \in \mathbb{Z} .
$$

Lemma B.16. Let $z \in \mathbb{C} \backslash \partial \mathbb{D}$. Then the functions $M_{\ell, \ell^{\prime}}(\cdot, k), \ell, \ell^{\prime}=0,1$, and $M_{ \pm}(\cdot, k), k \in \mathbb{Z}$, satisfy the following relations

$$
\begin{aligned}
& M_{0,0}(z, k)=1+\frac{\left[\overline{a_{k}}-\overline{b_{k}} M_{+}(z, k)\right]\left[a_{k}+b_{k} M_{-}(z, k)\right]}{\rho_{k}^{2}\left[M_{+}(z, k)-M_{-}(z, k)\right]}, \\
& M_{1,1}(z, k)=\frac{1-M_{+}(z, k) M_{-}(z, k)}{M_{+}(z, k)-M_{-}(z, k)}, \\
& M_{0,1}(z, k)=\frac{-1}{\rho_{k}\left[M_{+}(z, k)-M_{-}(z, k)\right]} \begin{cases}{\left[1-M_{+}(z, k)\right]\left[\overline{a_{k}}-\overline{b_{k}} M_{-}(z, k)\right],} & k \text { odd }, \\
{\left[1+M_{+}(z, k)\right]\left[a_{k}+b_{k} M_{-}(z, k)\right],} & k \text { even, } \\
\text { (B.62) }\end{cases} \\
& M_{1,0}(z, k)=\frac{-1}{\rho_{k}\left[M_{+}(z, k)-M_{-}(z, k)\right]} \begin{cases}{\left[1+M_{+}(z, k)\right]\left[a_{k}+b_{k} M_{-}(z, k)\right],} & k \text { odd, } \\
{\left[1-M_{+}(z, k)\right]\left[\overline{a_{k}}-\overline{b_{k}} M_{-}(z, k)\right],} & k \text { even },\end{cases}
\end{aligned}
$$

where $a_{k}=1+\alpha_{k}$ and $b_{k}=1-\alpha_{k}, k \in \mathbb{Z}$.

Finally, introducing the functions $\Phi_{1,1}(\cdot, k), k \in \mathbb{Z}$, by

$$
\Phi_{1,1}(z, k)=\frac{M_{1,1}(z, k)-1}{M_{1,1}(z, k)+1}, \quad z \in \mathbb{C} \backslash \partial \mathbb{D},
$$

then,

$$
M_{1,1}(z, k)=\frac{1+\Phi_{1,1}(z, k)}{1-\Phi_{1,1}(z, k)}, \quad z \in \mathbb{C} \backslash \partial \mathbb{D} .
$$

Lemma B.17. The function $\left.\Phi_{1,1}\right|_{\mathbb{D}}$ is a Schur function and $\Phi_{1,1}$ is related to $\Phi_{ \pm}$ by

$$
\Phi_{1,1}(z, k)=\frac{\Phi_{+}(z, k)}{\Phi_{-}(z, k)}, \quad z \in \mathbb{C} \backslash \partial \mathbb{D}, k \in \mathbb{Z} .
$$

Denoting by $I_{2}$ the identity operator in $\mathbb{C}^{2}$, we state the following result.

Theorem B.18. Let $k_{0} \in \mathbb{Z}$. Then the $C M V$ operator $U$ on $\ell^{2}(\mathbb{Z})$ is unitarily equivalent to the operator of multiplication by $I_{2}$ id (where $\left.i d(\zeta)=\zeta, \zeta \in \partial \mathbb{D}\right)$ on $L^{2}\left(\partial \mathbb{D} ; d \Omega\left(\cdot, k_{0}\right)\right)$. Thus,

$$
\sigma(U)=\operatorname{supp}\left(d \Omega\left(\cdot, k_{0}\right)\right)=\operatorname{supp}\left(d \Omega^{\operatorname{tr}}\left(\cdot, k_{0}\right)\right),
$$

where

$$
d \Omega^{\operatorname{tr}}\left(\cdot, k_{0}\right)=d \Omega_{0,0}\left(\cdot, k_{0}\right)+d \Omega_{1,1}\left(\cdot, k_{0}\right)
$$

denotes the trace measure of $d \Omega\left(\cdot, k_{0}\right)$.

Acknowledgments. We are indebted to Barry Simon for providing us with a copy of his forthcoming two-volume treatise [45] and for his interest in this paper. 


\section{REFERENCES}

[1] M. J. Ablowitz and J. F. Ladik, Nonlinear differential-difference equations, J. Math. Phys. 16, 598-603 (1975).

[2] M. J. Ablowitz, B. Prinari, and A. D. Trubatch, Discrete and Continuous Nonlinear Schrödinger Systems, London Math. Soc. Lecture Note Series, Vol. 302, Cambridge University Press, Cambridge, 2004.

[3] N. I. Akhiezer, The Classical Moment Problem, Oliver \& Boyd., Edinburgh, 1965.

[4] N. I. Akhiezer and I. M. Glazman, Theory of Operators in Hilbert Space, Vol. I, Pitman, Boston, 1981.

[5] N. Aronszajn and W. F. Donoghue, On exponential representations of analytic functions in the upper half-plane with positive imaginary part, J. Anal. Math. 5, 321-388 (1956-57).

[6] N. Aronszajn and W. F. Donoghue, A supplement to the paper on exponential representations of analytic functions in the upper half-plane with positive imaginary parts, J. Analyse Math. 12, 113-127 (1964).

[7] G. Borg, Eine Umkehrung der Sturm-Liouvilleschen Eigenwertaufgabe, Acta Math. 78, 1-96 (1946).

[8] M. J. Cantero, L. Moral, and L. Velázquez, Five-diagonal matrices and zeros of orthogonal polynomials on the unit circle, Lin. Algebra Appl. 362, 29-56 (2003).

[9] S. Clark and F. Gesztesy, Weyl-Titchmarsh $M$-function asymptotics and Borg-type theorems for Dirac operators, Trans. Amer. Math. Soc. 354, 3475-3534 (2002).

[10] S. Clark, F. Gesztesy, H. Holden, and B. M. Levitan, Borg-type theorems for matrix-valued Schrödinger operators, J. Diff. Eqs. 167, 181-210 (2000).

[11] S. Clark, F. Gesztesy, and W. Renger, Borg-type theorems for matrix-valued Jacobi and Dirac finite difference operators, preprint, 2004.

[12] W. Craig, The trace formula for Schrödinger operators on the line, Commun. Math. Phys. 126, 379-407 (1989).

[13] P. Deift and B. Simon, Almost periodic Schrödinger operators III. The absolutely continuous spectrum in one dimension, Commun. Math. Phys. 90, 389-411 (1983).

[14] B. Després, The Borg theorem for the vectorial Hill's equation, Inverse Probl. 11, 97-121 (1995).

[15] H. Flaschka, Discrete and periodic illustrations of some aspects of the inverse method, in Dynamical Systems, Theory and Applications, J. Moser (ed.), Lecture Notes In Physics, Vol. 38, Springer Verlag, Berlin, 1975, p. 441-466.

[16] J. S. Geronimo, F. Gesztesy, H. Holden, Algebro-geometric solutions of the Baxter-Szegö difference equation, to appear in Commun. Math. Phys.

[17] J. S. Geronimo and R. Johnson, Rotation number associated with difference equations satisfied by polynomials orthogonal on the unit circle, J. Diff. Eqs. 132, 140-178 (1996).

[18] J. S. Geronimo and R. Johnson, An inverse problem associated with polynomials orthogonal on the unit circle, Commun. Math. Phys. 193, 125-150 (1998).

[19] J. S. Geronimo and A. Teplyaev, A difference equation arising from the trigonometric moment problem having random reflection coefficients-an operator theoretic approach, J. Funct. Anal. 123, 12-45 (1994).

[20] J. Geronimus, On the trigonometric moment problem, Ann. Math. 47, 742-761 (1946).

[21] Ya. L. Geronimus, Polynomials orthogonal on a circle and their applications, Commun. Soc. Mat. Kharkov 15, 35-120 (1948); Amer. Math. Soc. Transl. (1) 3, 1-78 (1962).

[22] Ya. L. Geronimus, Orthogonal Polynomials, Consultants Bureau, New York, 1961.

[23] F. Gesztesy and H. Holden, Soliton Equations and Their Algebro-Geometric Solutions. Volume II: (1+1)-Dimensional Discrete Models, Cambridge Studies in Adv. Math., Cambridge University Press, Cambridge, in preparation.

[24] F. Gesztesy, M. Krishna, and G. Teschl, On isospectral sets of Jacobi operators, Commun. Math. Phys. 181, 631-645 (1996).

[25] F. Gesztesy and B. Simon, The $\xi$ function, Acta Math. 176, 49-71 (1996).

[26] F. Gesztesy and E. Tsekanovskii, On matrix-valued Herglotz functions, Math. Nachr. 218, 61-138 (2000).

[27] F. Gesztesy and M. Zinchenko, Weyl-Titchmarsh theory for CMV operators associated with orthogonal polynmials on the unit circle, preprint, 2004. 
[28] R. Giachetti and R. A. Johnson, Spectral theory of second-order almost periodic differential operators and its relation to classes of nonlinear evolution equations, Nuovo Cim. 82B, $125-168$ (1984).

[29] R. Giachetti and R. A. Johnson, The Floquet exponent for two-dimensional linear systems with bounded coefficients, J. Math. pures et appl. 65, 93-117 (1986).

[30] L. Golinskii and P. Nevai, Szegö difference equations, transfer matrices and orthogonal polynomials on the unit circle, Commun. Math. Phys. 223, 223-259 (2001).

[31] D. S. Greenstein, On the analytic continuation of functions which map the upper half plane into itself, J. Math. Anal. Appl. 1, 355-362 (1960).

[32] U. Grenander and G. Szegö, Toeplitz Forms and their Applications, University of California Press, Berkeley, 1958; 2nd ed., Chelsea, New York, 1984.

[33] R. A. Johnson, The recurrent Hill's equation, J. Diff. Eqs. 46, 165-193 (1982).

[34] I. S. Kac and M. G. Krein, R-functions-analytic functions mapping the upper halfplane into itself, Amer. Math. Soc. Transl. (2) 103, 1-18 (1974).

[35] S. Kotani, Ljapunov indices determine absolutely continuous spectra of stationary random one-dimensional Schrödinger operators, in Stochastic Analysis, K. Itǒ (ed.), North-Holland, Amsterdam, 1984, pp. 225-247.

[36] S. Kotani, One-dimensional random Schrödinger operators and Herglotz functions, in Probabilistic Methods in Mathematical Physics, K. Itǒ and N. Ikeda (eds.), Academic Press, New York, 1987, pp. 219-250.

[37] S. Kotani and M. Krishna, Almost periodicity of some random potentials, J. Funct. Anal. 78, 390-405 (1988).

[38] M. G. Krein, On a generalization of some investigations of G. Szegö, V. Smirnoff, and A. Kolmogoroff, Doklady Akad. Nauk SSSR 46, 91-94 (1945). (Russian).

[39] M. M. Malamud, Similarity of Volterra operators and related questions of the theory of differential equations of fractional order, Trans. Moscow Math. Soc. 55, 57-122 (1994).

[40] M. M. Malamud, Borg type theorems for first-order systems on a finite interval, Funct. Anal. Appl. 33, 64-68 (1999).

[41] P. D. Miller, N. M. Ercolani, I. M. Krichever, and C. D. Levermore, Finite genus solutions to the Ablowitz-Ladik equations, Comm. Pure Appl. Math. 4, 1369-1440 (1995).

[42] I. Nenciu, Lax pairs for the Ablowitz-Ladik system via orthogonal polynomials on the unit circle, Int. Math. Res. Notices, to appear.

[43] F. Peherstorfer and P. Yuditskii, Asymptotic behavior of polynomials orthonormal on a homogeneous set, J. Analyse Math. 89, 113-154 (2003).

[44] B. Simon, Analogs of the $m$-function in the theory of orthogonal polynomials on the unit circle, J. Comp. Appl. Math. 171, 411-424 (2004).

[45] B. Simon, Orthogonal Polynomials on the Unit Circle, Part 1: Classical Theory, Part 2: Spectral Theory, AMS Colloquium Publication Series, Vol. 54, Providence, R.I., 2005.

[46] B. Simon, Orthogonal polynomials on the unit circle: New results, Intl. Math. Res. Notices, 2004, No. 53, 2837-2880.

[47] M. Sodin and P. Yuditskii, Almost periodic Sturm-Liouville operators with Cantor homogeneous spectrum, Comment. Math. Helvetici 70, 639-658 (1995).

[48] M. Sodin and P. Yuditskii, Almost periodic Jacobi matrices with homogeneous spectrum, infinite dimensional Jacobi inversion, and Hardy spaces of character-automorphic functions, J. Geom. Anal. 7, 387-435 (1997).

[49] G. Szegö, Beiträge zur Theorie der Toeplitzschen Formen I, Math. Z. 6, 167-202 (1920).

[50] G. Szegö, Beiträge zur Theorie der Toeplitzschen Formen II, Math. Z. 9, 167-190 (1921).

[51] G. Szegö, Orthogonal Polynomials, Amer Math. Soc. Colloq. Publ., Vol. 23, Amer. Math. Soc., Providence, R.I., 1978.

[52] G. Teschl, Trace formulas and inverse spectral theory for Jacobi operators, Commun. Math. Phys. 196, 175-202 (1998).

[53] G. Teschl, Jacobi Operators and Completely Integrable Nonlinear Lattices, Mathematical Surveys and Monographs, 72, American Mathematical Society, (2000).

[54] Ju. Ja. Tomčuk, Orthogonal polynomials on a given system of arcs of the unit circle, Sov. Math. Dokl. 4, 931-934 (1963).

[55] S. Verblunsky, On positive harmonic functions: A contribution to the algebra of Fourier series, Proc. London Math. Soc. (2) 38, 125-157 (1935). 
[56] S. Verblunsky, On positive harmonic functions (second paper), Proc. London Math. Soc. (2) 40, 290-320 (1936).

Department of Mathematics, University of Missouri, Columbia, MO 65211, USA

E-mail address: fritz@math.missouri.edu

URL: http://www.math.missouri.edu/personnel/faculty/gesztesyf.html

Department of Mathematics, University of Missouri, Columbia, MO 65211, USA

E-mail address: maxim@math.missouri.edu 